\title{
Die Relevanz des Gruppendiskussionsverfahrens und der dokumentarischen Methode für die medienpädagogische Professionsforschung
}

\author{
Stefan Welling
}

\begin{abstract}
Ausgehend von der Notwendigkeit einer qualitativ-empirisch fundierten medienpädagogischen Professionsforschung erörtert der Beitrag, wie das Gruppendiskussionsverfahren für diesen Zweck nutzbar gemacht werden kann. Anhand der exemplarischen Rekonstruktion medienpädagogischer Orientierungsmuster von in der offenen Jugendarbeit tätigen Pädagogen/-innen mit Hilfe der dokumentarischen Methode wird die praktische Anwendung des Verfahrens demonstriert und kritisch reflektiert. Dabei wird u. a. deutlich, dass das Gruppendiskussionsverfahren gut geeignet ist, zur Schliessung existierender Lücken der medienpädagogischen Professionsforschung beizutragen und gleichzeitig einen vielversprechenden Ansatz zur Entwicklung einer regelmässig eingeforderten reflexiven Professionalisierung zu leisten vermag.
\end{abstract}

\section{Warum brauchen wir eine qualitativ-empirisch fundierte medienpädagogische Professionsforschung?}

Die Arbeit von Dieter Baacke gilt innerhalb der medienpädagogischen Fachdiskussion im deutschsprachigen Raum nach wie vor als zentraler Referenzpunkt. Medienpädagogik umfasst demnach die Gesamtheit der pädagogisch orientierten Beschäftigung mit Medien in Theorie und Praxis sowie alle «pädagogisch relevanten handlungsanleitenden Überlegungen mit Medienbezug» (Baacke 1997: 5). Darunter fallen «alle sozialpädagogischen, sozialpolitischen, und sozialkulturellen Überlegungen und Massnahmen sowie Angebote für Kinder, Jugendliche und Erwachsene, die ihre kulturellen Interessen und Entfaltungsmöglichkeiten, ihre persönlichen Wachstums- und Entwicklungschancen sowie ihre sozialen und politischen Ausdrucks- und Partizipationsmöglichkeiten betreffen» (ebd.). In den 1990er Jahren avancierte das Konzept der Medienkompetenz, das über weite Strecken auf einen, von einer kritisch-reflexiven Haltung getragenen, zweck- und zielgerichteten Mediengebrauch abhebt, zur Zieldimension medienpädagogischer Arbeit (Rein 1996, Schell 1999). Im Kontext der andauernden Mediatisierung aller gesellschaftlichen Bereiche, ertönt der Ruf nach der Vermittlung entsprechender Kompetenzen auf der Basis medienpädagogischer Praxis in anhaltender Lautstärke. Davon sind nahezu alle pädagogischen Berufe betroffen. Medienpädagogik ist 
dabei primär eine Querschnittsaufgabe, die innerhalb der verschiedenen Berufsfelder anteilig zum Tragen kommt. Die professionstheoretische Fundierung des medienpädagogischen Berufsbildes steht aber gleichwohl noch am Anfang (Hugger 2001, 2006, Neuss 2003).

Im vorliegenden Beitrag greife ich mit der Offenen Jugendarbeit exemplarisch einen pädagogischen Teilbereich heraus, in dem auch medienpädagogisch gearbeitet wird. Häufig sind es Sozialpädagogen/-innen mit unterschiedlich ausgeprägten Zusatzkompetenzen, aber auch nicht-pädagogisch qualifizierte Personen (z.B. Honorarkräfte), die diese Aufgaben übernehmen. Anhand von Beispielen aus einer qualitativ-empirischen Untersuchung zur computerunterstützten Jugendarbeit diskutiere ich die Eignung des Gruppendiskussionsverfahrens sowie der Auswertung der mit dem Verfahren erhobenen Daten mittels der dokumentarischen Methode für die medienpädagogische Professionsforschung aus primär methodisch-methodologischer Sicht (Welling 2007).

Generell dominieren symbolisch-interaktionistische, struktur- und systemtheoretische Ansätze die pädagogische Professionsforschung (Combe/Helsper 1996: 10-13, Schweppe 2003: 144). Alle drei verweisen auf einen Strukturkern professionellen Handelns, der vor allem durch Riskanz, Fehleranfälligkeit, Ungewissheit, Paradoxien und Antinomien charakterisiert ist (Schweppe 2003: 145, Hafeneger 2007: 14). Aufgrund dieser spezifischen Voraussetzungen lässt sich das professionelle Handeln weder wissenschaftlich steuern noch bürokratisch lenken bzw. expertokratisch aus allgemeinen Regelsätzen ableiten (Schweppe 2003: 145). Auf die Jugendarbeit übertragen, beruhen diese Paradoxien des professionellen Handelns, die allen Formen sozialer Arbeit inhärent sind, auf dem notwendigen Widerstreit divergierender Orientierungstendenzen bei der Bearbeitung der Probleme der Jugendlichen durch die Jugendarbeiter/innen (Schütze 1992, 2000). Letztlich sind es die unaufhebbaren Kernprobleme kommunikativer Interaktion und Welterkundung, die auch in diesem Feld pädagogischer Arbeit zum Tragen kommen (Schütze 2000: 50).

Um auf diese Problematik adäquat zu reagieren, müssen sich die Jugendarbeiter/innen aus strukturtheoretischer Professionssicht in die Situation ihrer Klientel hineinversetzen und deren Sinnhorizonte und Bewältigungsbedürfnisse adäquat erfassen, um parallel dazu auf Grundlage ihres Professionswissens stellvertretend geeignete Lösungen zu entwickeln (Tiefel 2004: 35). ${ }^{1}$ Voraussetzung dafür ist eine «doppelte Professionalisierung durch die wissenschaftliche Disziplin und die Praxis» (ebd., H.i.O.). Damit, so Hugger, ist zugleich das Grundproblem professionalisierten Handelns angesprochen, nämlich die zu erbringende Vermittlungsleistung zwischen Wissenschaft und Lebenspraxis bzw. zwischen Theorie und Praxis. Dabei

Für ein strukturtheoretisches Professionsverständnis steht insbesondere Oevermann (1996). Von ihm stammt auch das Konzept der stellvertretenden Deutung, das seit den 1980er Jahren die Professionalisierungsdiskussion in der Erziehungswissenschaft prägt (Hugger 2003: 45). 
zielt die Professionalisierung auf die Entwicklung einer Handlungsstruktur, die den professionell Handelnden ermöglichen soll, die lebenspraktischen Probleme ihrer Adressaten wissenschaftlich reflektiert zu bearbeiten, indem sie Fallanalysen und pädagogisches Handeln in ihrer beruflichen Praxis verbinden (Hugger 2003: 45). Auch Zacharias (2004: 167) betont die Bedeutung der in einem dialektisch-dialogischem Wechselverhältnis stehenden Theorie und Praxis für das pädagogische Handeln. Gleiches gilt für die Notwendigkeit des Reflektierens und Agierens sowie Denken und Machens. Dieser Wechsel zwischen Reflexion und Aktion ist auch für die medienpädagogische Praxis unverzichtbar, da sie in besonderer Weise auf Wirklichkeitskonstruktionen reagiert, die jenseits wissenschaftlicher Theorie-Konstruktionen im Verlauf der Alltagspraxis erfolgen (Baacke 1997: 5).

An dieser Stelle tun sich zwei erhebliche Probleme der medienpädagogischen Praxis auf: Erstens die Schwierigkeiten der Jugendarbeiter/innen, die Sinnhaftigkeit der adoleszenten Medienpraxis verstehend nachzuvollziehen und zweitens eine weit reichende Theorieferne der in der Jugendarbeit professionell Tätigen, die auch die medienpädagogische Arbeit tangiert. Neu sind diese Herausforderungen allerdings nicht: Bereits Ende der 1980er Jahre wiesen Böhnisch und Münchmeier (1987: 46) darauf hin, dass die Jugendlichen den Jugendarbeitern/-innen zunehmend fremd erscheinen und der jungen Generation und ihrem Handeln mit Unverständnis begegnen. Der Hinweis, dass die Jugendarbeiter/innen in Verdacht stünden, mittels vorgefertigter Zuschreibungen, eingespielter Deutungsmuster und Handlungsroutinen häufig gerade jene Schwierigkeiten und Problemfelder erst zu erschaffen oder zu verstärken, mit deren pädagogischer Bearbeitung sie sich dann beschäftigen, zeigt in die gleiche Richtung (King/Müller 2000: 11, Walser 2000: 251). Thole spricht in diesem Zusammenhang von expliziter Entprofessionalisierung und Dequalifizierungstendenzen in der Sozialpädagogik (Thole 2001, 2003). Eine systematische, wissenschaftlich gestützte und reflexive Fachlichkeit spielt demnach so gut wie keine Rolle für die Pädagogen/-innen bzw. dominiert eine «natürliche Fachlichkeit> ihre professionelle Praxis, die in der Regel nur auf einem populärwissenschaftlich und alltagspragmatisch fundierten Wissensrepertoire sowie vorrangig lebensweltlichen, biografisch angehäuften und alltagspraktischen Kompetenzen basiert (Thole 2001: 181f). In die gleiche Richtung zeigt eine aktuelle Untersuchung von Delmas und Scherr (2005), wonach sich die Praxis der Jugendarbeit in erheblicher Distanz zum Theoriediskurs entwickelt. Niesyto berichtet Ähnliches für die Medienarbeit, und die von ihm befragten Pädagogen/-innen verneinen grösstenteils eine Orientierung an bestimmten medienpädagogischen Modellen sowie die Berücksichtigung von Ergebnissen medienpädagogischer Forschung (Niesyto 2004: 30). Gleichzeitig macht ein Rest Nicht-Wissens und Nicht-Wissen-Könnens den Kern pädagogischen Handelns und pädagogischer Professionalität aus (Wimmer 1996: 425). Insofern ist Hugger (2001: 41f) zuzustimmen, der sich unter Be- 
zugnahme auf Wimmer explizit für die Anerkennung des Nicht-Wissens als Strukturmerkmal pädagogischer Professionalität ausspricht.

Den Versuch, die Gründe für die skizzierten Probleme mittels einer empirisch fundierten, gegenstandsangemessenen Auseinandersetzung mit dem interessierenden Gegenstand zu erklären, liegt nahe. Denn das Verstehen von Äusserungen oder Handlungen bzw. der ihnen impliziten Haltungen und Orientierungen setzt voraus, dass man die Alltagspraxis bzw. den Erlebniszusammenhang kennen gelernt hat, aus dem diese Äusserungen stammen (Bohnsack 2003: 59f). Bisher aber, so Schweppe (2003: 146), fehlen empirische Annäherungen an das berufliche bzw. professionelle Handeln von Sozialpädagogen/-innen weitgehend. Das gilt, von wenigen Ausnahmen abgesehen, analog für die medienpädagogische Forschung (Niesyto 2004, Welling/Brüggemann 2004).

Das Gruppendiskussionsverfahren und die dokumentarische Methode der Interpretation bieten sich als Instrumentarien für entsprechende Untersuchungen an, in deren Zentrum die oben hervorgehobenen, im Verlauf der Alltagspraxis erfolgenden Wirklichkeitskonstruktionen stehen. Diese Rekonstruktion zielt auf das der Praxis zugrunde liegende habitualisierte und teilweise inkorporierte Orientierungswissen, welches das Handeln relativ unabhängig vom subjektiv gemeinten Sinn strukturiert (Bohnsack u. a. 2001b: 9). Das bei der Arbeit mit der dokumentarischen Methode die empirische Basis des Akteurwissens nicht verlassen wird, unterscheidet sie von objektivistischen Zugängen, die nach Handlungsstrukturen shinter dem Rücken der Akteure» suchen (ebd.). Aufgrund der hohen Bedeutung der Handlungspraxis für die Vorgehensweise wird diese Form der Wissenssoziologie auch als praxeologisch bezeichnet, die sowohl die Praxis des Handelns wie diejenige des Sprechens, Darstellens oder Argumentierens anspricht (ebd.: 12f). Theorie- und Typenbildung erfolgen entsprechend auf der Grundlage der Rekonstruktion dieser Praxis bzw. des sie konstituierenden Erfahrungswissens (Bohnsack 2003: 10). Durch einen solchen Wechsel der Analyseeinstellung lassen sich auch die von Hugger angemahnten Schwächen einer theoriegeleiteten Typologisierung aufheben. Denn sie birgt reduktive Tendenzen einer Idealtypenbildung und vermag die ihr zugrunde liegende Wirklichkeit regelmässig nur eingeschränkt abzubilden (Hugger 2001: 55).

\section{Die Nutzung des Gruppendiskussionsverfahrens für die medienpädagogische Professionsforschung}

Der Einsatz von Gruppendiskussionen reicht im deutschsprachigen Raum bis in die 1950er Jahre zurück. ${ }^{2}$ Im Sinne der praxeologischen Wissenssoziologie kann man vom Gruppendiskussionsverfahren aber nur dort sprechen «wo die methodologische Bedeutung von Interaktions-, Diskurs- und Gruppenprozessen für die

2 Vgl. Loos und Schäffer (2001: 15ff) für eine Überblick zu den unterschiedlichen Ansätzen zur Arbeit mit Gruppendiskussionen. 
Konstitution von Meinungen, Orientierungs- und Bedeutungsmustern in einem zugrundliegenden theoretischen Modell, d. h. in metatheoretischen Kategorien mit theoriegeschichtlicher Tradition verankert sind» (Bohnsack 2000: 123). Diese Form des Gruppendiskussionsverfahren ist dabei, sich zu einem Standardverfahren der qualitativen Sozialforschung zu etablieren (Bohnsack u. a. 2006b: 6). Methodisch-methodologisch primär vor dem Hintergrund empirischer Jugendforschung fundiert (ebd.: 14), findet es inzwischen auch verstärkt Anwendung in der Medienund der medienpädagogischen Forschung. Dabei ist zuerst auf Burkhard Schäffer zu verweisen, der generationsspezifische Medienpraxiskulturen rekonstruiert und miteinander verglichen hat (Schäffer 2003). Des weiteren wird das Gruppendiskussionsverfahren auch im Rahmen der Arbeit des Hochschulartenübergreifenden Kompetenzzentrums für Genderforschung und Bildungsfragen in der Informationsgesellschaft (KGBI) eingesetzt. Untersucht wurden und werden dort insbesondere geschlechtsspezifische Aspekte von Computermedienpraxiskulturen Jugendlicher aus unterschiedlichen Bildungsmilieus (Buchen 2004, 2006, Buchen/Philipper 2002, Buchen/Straub 2006a, Straub 2005, 2006). Dabei werden auch medienpädagogische Themen behandelt, wie z. B. die Frage danach, welche Konsequenzen die den Computermedienpraxiskulturen zugrunde liegenden Orientierungsmuster für die schulische Medienbildung haben (Buchen/Straub 2006b, Buchen/Philipper 2002). In der pädagogischen Professionsforschung ist das Gruppendiskussionsverfahren bisher nur vereinzelt zum Einsatz gekommen (vgl. z. B. Krüger u. a. 2002 und Kutscher 2003). In der medienpädagogischen Professionsforschung spielt es so gut wie gar keine Rolle.

Die gegenwärtige theoretisch-methodische Fundierung und die Verbindung des Erhebungsverfahrens mit dem Analyseverfahren der dokumentarischen Methode gehen primär auf die Arbeit von und um Ralf Bohnsack herum zurück (Bohnsack 2003, Bohnsack u. a. 2001a, Bohnsack u. a. 2006a). Von zentraler Relevanz ist dabei die Konzeption des von Karl Mannheim entwickelten konjunktiven Erfahrungsraums, der das Kollektive theoretisch-methodisch in einer der empirischen Evidenz der Gruppendiskussion adäquaten Weise begründet (Bohnsack 2003: 108). Mannheim hat die dokumentarische Methode wissenssoziologisch geprägt und erkenntnistheoretisch begründet, die einen methodisch kontrollierbaren Zugang zu den konjunktiven Erfahrungen ermöglicht (Mannheim 1964 (1921)). Innerhalb konjunktiver Erfahrungsräume werden auf dem Wege habituellen Handelns grundlegende Orientierungen, Haltungen und Dispositionen erworben. Sie bilden den Grundstock eines habituellen 'Wissens wovon), das nicht oder noch nicht einer Ebene kommunikativ-generalisierten «Wissens worüber) entspricht (Schäffer 2001: 20). Ein konjunktiver Erfahrungsraum zwischen zwei Menschen zeichnet sich in diesem Sinne dadurch aus, dass beide über einen auf der vorsprachlichen Ebene angesiedelten gemeinsamen Pool verfügen, der neben Gesten und Körperhaltungen vor allem mit geteilten ästhetisch-kulturellen Ausdrucksformen gefüllt ist, 
in denen sich Grundintentionen und Gestaltungsprinzipien manifestieren. Neben dieser, quasi kleinsten Einheit konstituiert die gemeinsame Existenz in geistigen Beziehungen, wie sie sich z. B. durch die Arbeit im gleichen Berufsfeld ergibt, einen solchen Erfahrungsraum der daran beteiligten Subjekte auf der Grundlage gemeinsamer Praxis jenseits theoretischen Erkennens und kommunikativer Absichten (Bohnsack 2003: 62).

Man kann in diesem Kontext auch vom Milieu als konjunktiven Erfahrungsraum sprechen (ebd.: 11). Solche Milieus bilden sich im Verlauf der alltäglichen kollektiven Handlungspraxis, ihre Angehörigen verstehen sich im Medium des Selbstverständlichen. Dazu bedienen sie sich so genannter konjunktiver Begrifflichkeiten, die in ihrem umfassenden Gehalt nur denjenigen verständlich sind, die den Erlebnis- und Erfahrungszusammenhang, aus dem die Begrifflichkeiten stammen, kollektiv-biografisch teilen, d.h. diese Begrifflichkeiten sind indexial. Die Milieus sind untrennbar mit den in diesen Erfahrungsräumen hervortretenden Praxen verbunden und werden von diesen begründet (Schäffer 2003: 27). Unter anderem verbinden Gemeinsamkeiten des biografischen Erlebens oder der Sozialisationsgeschichte und strukturidentische Erlebnisse die Milieuangehörigen miteinander (Bohnsack 1998b: 97). Die Angehörigen der gleichen Profession sind aufgrund vergleichbarer beruflicher Sozialisationsmuster, strukturidentischer Erfahrungen aus der Arbeitspraxis und der Auseinandersetzung mit gleichen und ähnlichen Orientierungsschemata eng miteinander verbunden, sodass man auch von einem professionellen Milieu sprechen kann.

Die zentrale Prämisse bei der Durchführung von Gruppendiskussionen ist die Herstellung von Selbstläufigkeit. Diese zielt darauf ab, dass den Diskutierenden die Möglichkeit eingeräumt wird, ihre Orientierungsrahmen im Gespräch zu artikulieren. Erst wenn sich die Diskussion einer Gruppe in ihrer Eigenständigkeit entfalten kann, kommen die Relevanzsysteme ihrer Mitglieder zum Vorschein. Gleichzeitig wird auf diese Weise verhindert, dass in erster Linie die theoretisch induzierten Relevanzsysteme der Forschenden, z, B. in Form von mehr oder weniger offenen bzw. geschlossenen Diskussionsleitfäden, von den Gruppendiskussionsteilnehmern/innen abgearbeitet werden (Loos/Schäffer 2001: 52). ${ }^{3}$

Ausserdem erhalten die Diskussionsteilnehmer/innen aufgrund der multilateralen Interaktionsmöglichkeiten während der Gruppendiskussion weitaus grösseren Einfluss auf den Verlauf und die Themenhierarchie der Erhebung als beim Einsatz anderer Erhebungsinstrumente, da sich die feldspezifische Kommunikation zwischen Forschenden und Teilnehmenden alltagsnah und nicht-hierarchisch insze-

3 Der Grad der hergestellten Selbstläufigkeit lässt sich methodisch kontrollieren, indem anhand der Transkripte zwischen Sequenzen unterschieden wird, in denen die Diskussionsteilnehmerlnnen explizit auf die Interviewenden reagieren und solchen, in denen sie sich metaphorisch und dramaturgisch immer mehr steigern und die Interviewenden tendenziell in den Hintergrund treten (Loos/ Schäffer 2001: 52). Vgl. zur Durchführung von Gruppendiskussionen auch Loos und Schäffer (2001: 48ff) und Bohnsack (2003: 20ff). 
nieren lässt (Kutscher 2006: 189f). Das dient auch der methodischen Kontrolle der Erhebung, denn indem die Erforschten Gelegenheit erhalten ihr kommunikatives Regel- und Relevanzsystem zu entfalten, wird die Kontrolle zwischen den «Sprachen v von Forschenden und Erforschten über die Differenzen ihrer Interpretationsrahmen und ihrer Relevanzsysteme erst möglich (Bohnsack 2005: 69f).

Die dokumentarische Methode ist darauf gerichtet, «einen Zugang zum konjunktiven Wissen als dem je milieuspezifischen Orientierungswissen zu erschliessen» (Bohnsack u.a. 2001b: 14). Die Beziehung zum kollektiven Charakter der untersuchten Fälle steht dabei von Beginn an im Zentrum der Untersuchung. Das unterscheidet das Gruppendiskussionsverfahren auch in besonderer Weise von individuierenden Erhebungsverfahren wie z.B. dem narrativen Interview, bei dem die Beziehung zum kollektiven Charakter der untersuchten Fälle erst nachträglich hergestellt wird.

Im Rahmen der formulierenden Interpretation als erstem Analyseschritt werden zunächst eine thematische Struktur des vorliegenden Materials erstellt und Passagen ausgewählt, die zum Gegenstand der reflektierenden Interpretation werden sollen. Die Auswahl orientiert sich an der thematischen Relevanz der Passagen für die Ausgangsfragestellung sowie der thematischen Vergleichbarkeit mit Passagen aus anderen Diskussionen (Bohnsack 2003: 135). ${ }^{4}$

Die reflektierende, bzw. dokumentarische Interpretation des Forschungsmaterials ist der zweite und zentrale Schritt der dokumentarischen Methode. Im Fokus der methodologischen Vorgehensweise stehen die Rekonstruktion und die Exemplifizierung der Orientierungsmuster und des Orientierungsrahmens (der auch als Habitus bezeichnet werden kann), innerhalb dessen bestimmte Themen (z. B. der Umgang mit Computerspielen) abgehandelt werden (ebd.). ${ }^{5}$ Im Zuge der reflektierenden Interpretation vollzieht sich methodisch ein Wechsel von den 〈Was-> zu den 〈Wie-Fragen`: Was dokumentiert sich in dem, wie etwas gesagt wird, über den dahinter stehenden konjunktiven Erfahrungsraum bzw. die kollektive Handlungspraxis? An dieser Stelle kommen die Orientierungsmuster, bestehend aus Orientierungsschemata und Orientierungsrahmen zum Tragen. Die Orientierungsschemata umfassen institutionalisierte und damit normierte Ablaufmuster oder Erwartungsfahrpläne (z. B. verbindliche Projektziele oder jugendschutzrechtliche Vorgaben) genauso wie Handlungsentwürfe, an denen das Handeln im Sinne von (zweckrationalen) «Um-Zu-Motiven` orientiert ist (Bohnsack 1997b, 2001: 229f). Die

4 Vgl. Bohnsack und Nohl (2001) sowie Bohnsack (2003: 212ff) zur Durchführung der formulierenden und reflektierenden Interpretation.

5 Im Unterschied zu Hypothesen, mit denen ex ante formuliertes theoretisches Wissen an den Untersuchungsgegenstand herangetragen wird und «die den Charakter gegenstandsbezogener Theorien haben, stehen in der qualitativen Sozialforschung Meta-Theorien oder formale Theorien am Anfang des Forschungsprozesses». Begriffe wie der des Erfahrungsraumes, der Gruppe oder des Kollektives, genauso wie der des Orientierungsmusters und Orientierungsrahmens sowie die Kategorien der Diskursorganisation und der Dramaturgie des Diskurses bilden den meta-theoretischen Analyserahmen der dokumentarischen Methode (Bohnsack 2005: 70). 
Enaktierung, d.h. die handlungspraktische Umsetzung dieser Schemata, vollzieht sich im Medium der konjunktiven Erfahrung und des habituellen Handelns. Im Zusammenspiel dieser beiden Aspekte ist der übergreifende Orientierungsrahmen einer Gruppe angesiedelt (Bohnsack 1997b: 57, 1998b: 112). Die Orientierungsrahmen bilden sich im Sinne habitualisierter Wissensbestände dort, «wo diese (grundlegend kollektiven) Wissensbestände nicht nur internalisiert, sondern auch inkorporiert, d. h. in den modus operandi der körperlichen und sprachlichen Praktiken eingeschrieben und in diesem Sinne «mimetisch) angeeignet werden» (Bohnsack 2003: 132).

Die tiefergehenden semantischen Gehalte einer Gruppendiskussion lassen sich aber nur dann erfassen, wenn deren formale Struktur im Sinne der Diskursorganisation, d. h. die formale Bezugnahme der an den Diskussionen beteiligten Personen, zuvor verstehend erfasst wurde (Bohnsack/Przyborski 2006: 235). Erst diese Rekonstruktion erlaubt vailde Aussagen über die Existenz konjunktiver Erfahrungsräume oder Milieus und darüber, welcher Art die sie konstituierenden Gemeinsamkeiten sind. ${ }^{6}$ Indem die Existenz konjunktiver Erfahrungsräume nicht vorab durch Hypothesen und theoretische Relevanzsetzungen der Forschenden unterstellt werden, wird ein offener und in diesem Sinne rekonstruktiver Zugang zum Forschungsfeld eröffnet (ebd.: 246). Vor dem Hintergrund des Wissens um die natürlichen Standards der Erforschten, d. h. den im Forschungsfeld immer schon vorfindbaren Regeln, formalen Strukturen und ‘Standards〉 der Kommunikation und Darstellung, lassen sich auch die Interventionen der Forschenden methodisch kontrollieren und entsprechend strukturieren (ebd.: 247). Um die natürlichen Standards aber nicht lediglich zu übernehmen, sondern in gleicher Weise zu kontrollieren, muss ausserdem ein «Bruch» mit den Vorannahmen des common sense erfolgen, indem die in der Praxis alltäglicher Verständigung implizierten kommunikativen Regeln empirisch rekonstruiert werden (Bohnsack 2005: 72). ${ }^{7}$

Entscheidend für die Erschliessung der Orientierungsmuster ist, dass dem Rahmen und der Selektivität (die spezifische Weichen- und Problemstellung bei der Behandlung eines Themas) einer Gruppe Alternativen gegenüber gestellt werden, wie sie von anderen Gruppen bei vergleichbaren Themen verwendet werden (Bohnsack 2003: 136). Die Vergleichsgruppenbildung bzw. komparative Analyse ist zentral für das rekonstruktive Verfahren, denn erst durch den Kontrast in den Gemeinsamkeiten, d.h. dem Vergleich von Gruppen, die sich vor ein gemeinsames

6 Vgl. zur Diskursanalyse der dokumentarischen Methode, den Modi der Diskursorganisation und der Repräsentation von Erfahrungsräumen Przyborski (2004).

7 Diese common-sense Theorien adressieren die Ebene des kommunikativ-generalisierenden Wissens. Kommunikatives Wissen bzw. Erfahrungsräume basieren auf Verständigungsprozessen auf einer Ebene intendierten Handelns, die sich problemlos intersubjektiv überprüfen lassen (z. B. die Frage danach, ob ein Projekt Spass gemacht hat). Den ¿Gegenpolı bildet das konjunktive Wissen. Siehe zu den Theorien des common sense auch Bourdieu (1996: 278). 
entwicklungstypisches Problem gestellt sehen, werden im Zuge der unterschiedlichen Strategien der Problembewältigung milieutypische Unterschiede oder Kontraste sichtbar (Bohnsack 1989: 346). Gleichzeitig trägt diese Form des Vergleichs ebenfalls zur Kontrolle der Standortgebundenheit der Forschenden bei. Diese lässt sich zwar prinzipiell nie vollständig auflösen, durch die sukzessive Ersetzung der von den Forschenden an den Forschungsgegenstand herangetragenen impliziten Vergleichshorizonte durch aus dem empirischen Material rekonstruierte Vergleichsfälle lässt sich die Kontrolle aber zumindest methodisieren (Bohnsack 2001: 235f). Negative und positive Vergleichs- bzw. Gegenhorizonte sowie deren Enaktierungspotenziale sind wesentliche Komponenten des Erfahrungsraums einer Gruppe, die auch deren Rahmen konstituieren. Neben dem fallübergreifenden wird der fallinterne Vergleich bemüht, der durch das Gerüst der jeweiligen Rahmenkomponenten strukturiert wird. Indem man die jeweiligen Fälle vor dem Gegenhorizont anderer Fälle in ihre Bedeutungsschichten zerlegt, gelangt man zur Typenbildung (Bohnsack 2003: 141ff).

Dabei werden zum einen Bezüge zwischen spezifischen Orientierungen herausgearbeitet. Zum anderen zielt die Typenbildung auf die Erlebnis- oder existenzielle Hintergründe, in dem die Entstehung der biografischen Orientierungen zu suchen ist (ebd.: 141). Anders als die Typenbildung des common sense, wie man sie in prominenter Form bei dem vom Max Weber vorgeschlagenen Idealtypus findet, zielt die praxeologische Typenbildung darauf ab, die Um-zu-Motive im Zusammenhang der sie konstituierenden Bedingungen zu erfassen, d. h. jenen Erlebniszusammenhängen, aus denen sie entstanden sind (den Weil-Motiven) (Bohnsack 2001: 227, 2003: 145f). Insofern ist diese Form der Typenbildung auch nicht mit einer Typisierung von Ergebnissen im Sinne einer Zusammenfassung und Kategorisierung von Aussagen und deren Interpretationen zu verwechseln. Vielmehr folgt sie den der komparativen Analyse zugrunde liegenden Dimensionen des existenziellen Hintergrunds (z. B. Geschlechts- oder Bildungsmilieuzugehörigkeit), aus denen heraus sich die einzelnen Fälle (repräsentiert durch die jeweiligen Gruppen) erklären (Loos/Schäffer 2001: 71f). Die Generierung einer validen Typenbildung setzt voraus, dass die verschiedenen Typiken entlang der unterschiedlichen Dimensionen oder Erfahrungsräume eines Falles herausgearbeitet werden, sodass sich an diesem unterschiedliche Typiken überlagern (Bohnsack 2003: 143). Denn milieutypische Unterschiede werden gerade dann konturiert sichtbar, wenn sie vor dem Hintergrund von Gemeinsamkeiten (z. B. gemeinsames entwicklungstypisches Phänomen) beobachtet werden können. Somit ist der Kontrast in der Gemeinsamkeit (s. o.) auch das fundamentale Prinzip bei der Generierung einzelner Typiken und zugleich die Klammer, die eine ganze Typologie zusammenhält (ebd.). Gleichzeitig ist die Typenbildung für die qualitative Sozialforschung unter dem Aspekt der Generalisierbarkeit ihrer Ergebnisse unverzichtbar (Bohnsack 2005: 76f). 


\section{Exemplarische Rekonstruktion medienpädagogischer Orientierungsmuster}

Die folgende exemplarische Rekonstruktion medienpädagogischer Orientierungsmuster erfolgt auf der Basis von drei Gruppendiskussionen mit Jugendarbeiter/innen, die im Rahmen von Welling (2007) geführt wurden. ${ }^{8}$ Diese Arbeit befasst sich mit den Potenzialen einer milieusensitiven computerunterstützten Jugendarbeit. Der Milieuaspekt adressiert zwei wechselseitig verschränkte Perspektiven: Einerseits die Milieubildung als Analysedimension, zum anderen als (medien-)pädagogische Gestaltungsdimension (Bohnsack 1998b). Dabei geht es u. a. um die Frage, welchen Anteil die Computermedien an der intergenerationellen Praxis zwischen den Jugendarbeitern/-innen und den Jugendlichen in den Jugendzentren haben, was für Erwartungen beider Gruppen diesen Praxen zugrunde liegen und welche Konsequenzen deren Verlauf für die Prozesse der Medienbildung hat? Medienbildung adressiert dabei die Resultate der Mediennutzung aus der Perspektive der Ermöglichung respektive Verhinderung von Bildungsprozessen (Pietrass 2002). Bildung als biografischer Wandlungsprozess (Schütze 1994) zielt in diesem Zusammenhang auf die Veränderung der Selbst- und Weltreferenzen der daran beteiligten Subjekte ab (Marotzki 1990). Ein Zugewinn an Wissen im Sinne von Medienkompetenz ist dabei nicht auszuschliessen, tritt aber nicht in jedem Fall ein, und auch der vermeintlich inkompetente Umgang mit Medien kann in diesem Sinne durchaus bildend sein.

Ich beginne mit einem Ausschnitt aus der Gruppe Traube, die aus vier Frauen besteht, die seit mehreren Jahren in Kinder- und Jugendeinrichtungen arbeiten und rund viereinhalb Monate vor der Diskussion an einem mehrwöchigen medienpädagogischen Qualifizierungsangebot für Anfängerinnen teilgenommen haben. ${ }^{9}$ In den Diskussionen nimmt die Auseinandersetzung mit der gerade bei jüngeren Jugendlichen sehr populären Praxis des Chattens grossen Raum ein. Dabei treten die Jugendlichen mit Hilfe entsprechender Software in synchrone computervermittelte Kommunikation. Diese Interaktionen finden meistens innerhalb so genannter Communities statt, zu denen man in der Regel durch Registrierung Zugang erhält. Mit einem so genannten Nickname kann man sich dann in den verschiedenen Chatrooms der jeweiligen Community anmelden und mit Anderen in unterschiedlichen Gruppenkonstellationen interagieren. Auch in den Jugendzentren erfreut sich das Chatten grosser Beliebtheit. Obwohl die Praxis durchaus bildungsrelevant ist, stösst sie bei vielen Jugendarbeiter/innen auf Unverständnis und Ablehnung (Brüggemann/Welling 2006). Das gilt auch für die Gruppe Traube. Nachdem die Frauen bereits mehrfach das Chatten angesprochen haben, fragt sie der Intervie-

\footnotetext{
8 Im Rahmen der Untersuchung wurden insgesamt sechs Gruppendiskussionen mit Jugendarbeiterlnnen und 12 mit Jugendlichen geführt.

9 Die Gruppendiskussion wurde im Winter 2005 geführt. Die Frauen sind im Alter zwischen Ende 30 und Ende 40
} 
wer, ob sie im Kontext dieser Praxis Anknüpfungspunkte für ihre Arbeit sehen. Die Ausführungen der Frauen konzentrieren sich zunächst auf die gelegentliche Kontrolle der Praxis und das Intervenieren beim Verdacht auf jugendschutzrechtlich bedenkliche Kommunikationsverläufe. In der Einrichtung der Jugendarbeiterin Dagmar darf an einigen Computern gar nicht gechattet werden (Passage Umgang mit dem Chatten). ${ }^{10}$

Df: Aber da, in dem Raum haben wir gesagt, ist Chat-Verbot. Weil das sind Arbeits-PCs, habe ich denen damals erzählt. Da können wir tolle Sachen mit machen. Und chatten könnt ihr vorne. Weil wenn ich die auch zum Chatten freigebe, denn wär da nur noch so'n Tohuwabohu. Dann würden die Mädchen, die sonst gar nicht in der Mädchengruppe sind, würden da rein wollen, um da zu chatten. Und denn sag ich immer nö, das ist um irgendwelche schönen Sachen zu machen

Bf: Also, das einzige, ich finde, was da von Vorteil ist, ist dass sie da eben so schnell Schreiben lernen und

Df: $\quad \square$ Mmmh

Bf: und so was halt. Da wüsste ich sonst keine Anknüpfungspunkte, was man da überhaupt so. Es sei denn, man verfolgt vielleicht mal was, was die chatten, um zu merken, och, das interessiert die, wenn sie mal so was schreiben sollten. Wenn das nicht nur so, oach. Manchmal ist das ja nur so, da denkst du, o::h Gott

Df: Also, ich hab das höchstens hinterher mal so im Gespräch. Oh, da war einer im Chat und so. Wenn die Mädchen sich unterhalten. Das ich denn mal so nachfrage. Wie? Ist der blöd, oder so. Was will der überhaupt? Oach, meine Handynummer oder dies. Das man dann sagt, z.B. das machst du ja wohl nicht oder dies. Aber, so direkt beim Chatten, da, das wollen die auch nicht

Bf: Die beschimpfen sich auch da manchmal ganz also, was die auch gar nicht so empfinden. Aber immer (.....), Ja, wieso, das machen alle da. Also ganz

$\square$ Die haben auch teilweise eine ganz

$\square$ komische Ausdrücke Dganz

eigenartige Sprache. Wenn ich da meinem Sohn mal so über die Schulter guck, ne. Ich sag, sag mal, welche Klasse bist du. Erste Klasse, http://klippklang.ch/lehrmittel/index.htm oder was? Die schreiben ein Deutsch (.) So, ich kann das jetzt gar nicht wiedergeben. Das sind alles so Kürzel

10 Bei der Transkription kommen die im Zuge des Gruppendiskussionsverfahrens etablierten Transkriptionsrichtlinien zum Einsatz. Unterstreichungen im Text verweisen auf Betonungen. Der rechte Winkel vor Wörtern markiert den Beginn von Wortüberlappungen. Begriffe zwischen zwei @-Zeichen sind lachend gesprochen, ein Punkt in Klammer markiert ein kurzes Auflachen. Punkte in Klammern verweisen auf unverständlich gesprochene Worte, die nicht transkribiert werden konnten. Vgl. auch Bohnsack (2003: 235f). 


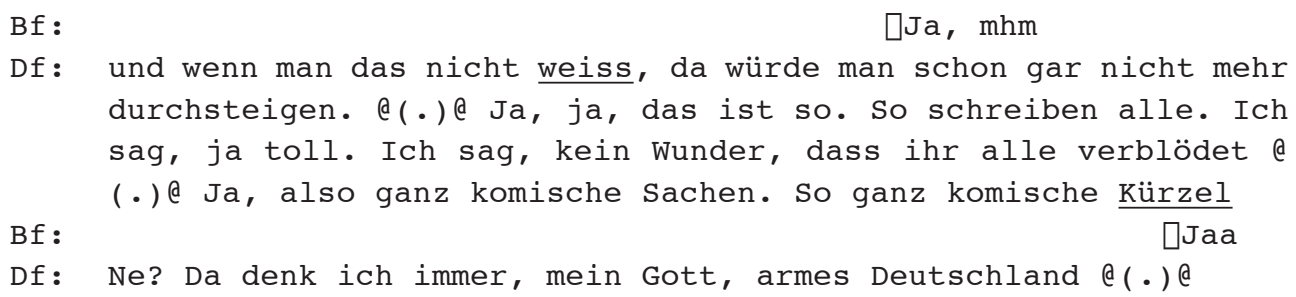

Den Jugendlichen wird das Chatten in einem der Räume mit der Begründung verwehrt, dass das «Arbeits-PC's» seien, an denen man «tolle» Sachen machen könne. Nur die zweckrationale und ernsthafte Nutzung, stellt die Entwicklung einer attraktiven Praxis in Aussicht bzw. wird suggeriert, dass diese Praxis grossartige und unglaubliche Resultate hervorzubringen vermag. Das Chatten konstituiert damit einen negativen Gegenhorizont zur berufsbiografisch orientierten Nutzung der Computermedien, für die der Begriff der Arbeit synonym steht. Mit dem Fortgang der Erklärung der Jugendarbeiterin Dagmar wird ein weiterer Grund für die Suspendierung der Medienpraxis offenbar. Würde sie selbige auch in dem besagten Raum zulassen, hätte das zur Folge, dass dort «nur auch so'n Tohuwabohu» entstünde. Der Verweis auf die arbeitsame Praxis erweist sich insofern als Vorwand, um eine unkontrollierte und spontane Praxis zu verhindern. Diese Logik entspricht dem im Verlauf der antizipierten Computermedienpraxis immer wieder vorgebrachten Wunsch nach Sicherheit und Ordnung. Die Suche nach Sicherheit resultiert aus der hohen Unsicherheit der Jugendarbeiterinnen im Umgang mit den Computermedien und der daraus rührenden Angst, dass es im Verlauf der medienpädagogischen Arbeit zu unvorhersehbaren Schwierigkeiten kommen könnte, die sich nicht beheben lassen und Gefühle von Frustration und Verzweiflung hervorrufen. Solche Situationen werden im Verlauf der Gruppendiskussion immer wieder artikuliert. Die Suche nach Sicherheit wird vom Wunsch nach Ordnung im Sinne von Übersichtlichkeit begleitet, die geeignet scheint, ein ausreichendes Mass an Kontrolle über die medienpädagogische Arbeit zu gewinnen. Wo die Praxis unsicher ist und mehr Ordnung gewünscht wird, bietet sich der Versuch, die Spontaneität der Praxis zu reduzieren als probate Umsetzungsmöglichkeit an. Gleichzeitig erweisen sich solche, von Spontaneität und aktionistischem Handeln geprägte Situation gerade für Jugendliche aus so genannten bildungsfernen Milieus als hochrelevant für die Initiierung von Bildungsprozessen (Bohnsack 1997a, Nohl 2006).

Die Anschlussproposition der Jugendarbeiterin Brigitte macht deutlich, dass auch sie dem Chatten gegenüber skeptisch eingestellt ist. Als einzigen Vorteil der Praxis sieht sie, dass die Mädchen lernen, schnell am Computer zu schreiben. Wieder sind es ausschliesslich instrumentelle Kompetenzen, die im Zuge der Auseinandersetzung mit der Praxis positiv zum Tragen kommen. Sie kann daher ad hoc keine "Anknüpfungspunkte» für ihre pädagogische Arbeit entdecken bzw. kann sie auf keine korrespondierenden Praxiserfahrungen rekurieren. Nur im Gedankenexpe- 
riment erschliesst sich eine Möglichkeit, die Praxis der Mädchen zum Gegenstand der medienpädagogischen Arbeit zu machen, indem man versuchen könnte zu verfolgen, was Gegenstand der Kommunikation im Chat ist, um so mehr über die Interessen der Jugendlichen zu erfahren, $d$. h. was für sie von biografischer Relevanz ist. Das würde aber voraussetzen, dass die Jugendlichen Texte erstellen, die solche Rückschlüsse zulassen. Das scheint aber nicht der Fall zu sein, vielmehr erweist sich die Kommunikation im Chat, wenn Brigitte ihr einmal Gewahr wird, als unverständlich, sodass im übertragenen Sinne nur eine überirdische Instanz ("Gott») den Verständnisprozess ermöglichen könnte bzw. für sie eine entsprechende Perspektivenübernahme unmöglich ist. Diese Stelle veranschaulicht exemplarisch in prägnanter Weise die in Kapitel 1 angesprochenen divergierenden Orientierungstendenzen.

Indirekt verifiziert Dagmar die Proposition von Brigitte. Wenn es überhaupt eine Möglichkeit gibt, die Kommunikation im Chat zum Gegenstand der eigenen Arbeit zu machen, dann im Kontext von Anschlusskommunikation, wenn sich die Mädchen über ihre Erlebnisse und Begegnungen mit Dritten im Chat unterhalten. In solchen Situationen versucht Dagmar mittels Nachfragen etwas über die Interaktionsverläufe der Mädchen zu erfahren. Man könne z. B. fragen, ob eine Person «blöd» sei und was sie von den Mädchen wollte. Der Gegenstand der Kommunikation erscheint zunächst undurchsichtig. Wenn die Mädchen aber eine solche Frage mit dem Hinweis auf ihre Mobiltelefonnummer beantworten, ist klar, dass es sich um einen Versuch gehandelt hat, den Kontakt im virtuellen Raum in eine Praxis unter Anwesenden zu überführen. Dann kann man die Mädchen ermahnen, solche Informationen nicht weiterzugeben. In solchen Situationen steht nicht das Interesse an der biografischen Relevanz des Chattens im Zentrum der professionellen Praxis, sondern die Orientierung an der zu übernehmenden Verantwortung für das Handeln der Jugendlichen, die einen zentralen Anteil an den professionellen Orientierungen der Jugendarbeiter/innen hat (Welling/Brüggemann 2004: 56f). Auch dabei tun sich Widersprüche auf. Einerseits ist zu fragen, welche rechtliche Verantwortung die Mitarbeiter/innen (i.S.v. Haftung und Einhaltung der Bestimmungen des Kinder- und Jugendschutzes) für das Handeln der Kinder und Jugendlichen übernehmen müssen. Zum anderen ist zu berücksichtigen, welche persönliche Verantwortung die Pädagogen/-innen für das Handeln der Kinder und Jugendlichen zu haben glauben.

Schliesslich, so Dagmar, wollen die Mädchen nicht, dass sich die Jugendarbeiterinnen in die Chatpraxis einmischen. Gleichzeitig widerstrebt es ihnen auch, die Praxis der Jugendlichen zu kontrollieren (ebd.). Da das Chatten die Medienpraxis in den Kinder- und Jugendeinrichtungen aber über weite Strecken prägt, entzieht es sich der Wahrnehmung der Jugendarbeiter/innen nicht vollständig. So berichtet Brigitte, dass sich die Mädchen im Chat gelegentlich gegenseitig beschimpfen, das aber «nicht so empfinden». Entscheidend für die Bewertung dieser Schmä- 
hungen ist nicht der, auf der kommunikativen Sinnebene liegende diffamierende Gehalt, sondern deren konjunktive Bedeutung, die auf geteilte biografische oder strukturidentische Erfahrungen zurückgeht. Die Beschimpfungen haben somit rituellen Charakter und dienen dem Gruppenzusammenhalt («machen alle») (vgl. auch Zirfas/Wulf 2001). Ohne es zu ahnen, hält Brigitte mit dieser ansatzweisen Perspektivenübernahme den Schlüssel für die intergenerationelle Verständigung in den Händen. An dieser Stelle beginnt sich zugleich die Gruppendiskussion von der Fragestellung des Interviewers zu entfernen und gewinnt an Selbstläufigkeit. Brigitte kommt über den geschilderten Ansatz nicht hinaus, die von den Jugendlichen verwendeten Ausdrücke erscheinen ihr «komisch», d. h. sonderbar und wunderlich. Dagmar wartet nicht, bis Brigitte ihre Beschreibung beendet hat und fällt ihr ins Wort. Gemeinsam erarbeiten sie, dass es nicht nur einzelne Ausdrücke sind, die ihnen unverständlich bleiben, sondern die gesamte Kommunikation, die eine eigenständige und zugleich «eigenartige» Sprache konstituiert. Dagmar bewertet diese Sprache anhand externer Massstäbe, die aus der schulischen Beurteilungspraxis rühren und unterstellt ihrem Sohn, dass seine Kommunikation im Chat, gemessen an ihrer orthografischen und grammatikalischen Korrektheit, dem schriftsprachlichen Leistungsvermögen eines Erstklässlers entspricht. Ihr Sohn wird quasi zum Stellvertreter der Jugendlichen, da die Mädchen, wie oben geschildert, nicht wollen, dass sich die Jugendarbeiterin in die Chatpraxis einmischt. Dass es sich bei den angesprochenen Kürzeln um Bestandteile einer eigenen, dem Chat angemessenen Sprache handelt, die auf vielerlei Abkürzungen und Verkürzungen basiert, um in kürzester Zeit möglichst viel mitteilen zu können, bleibt unberücksichtigt bzw. tritt hinter die Orientierung an der formalen Beurteilung zurück. Gleichwohl erkennt Dagmar an, dass man auch diese Sprache erlernen muss, um sie zu verstehen. Dieser Schritt bleibt aber aus, da sie dieser Sprache keinen Wert zuerkennt. Auch der Hinweis ihres Sohnes, dass sich alle chattenden Jugendlichen dieser Ausdrucksformen bedienen, trägt nicht zur Akzeptanzförderung bei. Im Gegenteil würde das viel mehr erklären, warum sie alle «verblöden», sodass die Praxis des Chattens nicht nur wertlos, sondern auch der kognitiven Entwicklung abträglich ist. Anschliessend wiederholt Dagmar noch einmal, wie sonderbar und wunderlich ihr diese Form der Kommunikation erscheint. Ihre Ausführungen werden mehrfach von Brigitte bestätigt, die damit die Strukturidentität der Erfahrung verifiziert. Abschliessend stilisiert Dagmar die kritisierte Kommunikationsform und die antizipierten Folgen in Form einer Zwischenkonklusion zur nationalen Problemlage («armes Deutschland»), d. h. die Praxis ist nicht nur in ihrer individuellen Ausprägung bedenklich, sondern auch gesellschaftlich fragwürdig. Abermals bleibt als Lösung nur das Anrufen einer übersinnlichen Einflussgrösse («mein Gott») bzw. verweist die Transzendierung der Problematik auf fehlende Ansätze der Bearbeitung auf Basis der eigenen berufsbiografischen Orientierungen. Eine pädagogisch motivierte Auseinandersetzung mit der Praxis spielt über den Aspekt der zu über- 
nehmenden Verantwortung hinaus für die Pädagoginnen keine Rolle und scheint zudem an der umfassenden Fremdheit gegenüber der Medienpraxis der Jugendlichen zu scheitern.

In der Gruppendiskussion mit den medienpädagogisch erfahreneren Jugendarbeiter/innen der Gruppe Kirsche spielt die in der Gruppe Traube aufgeworfene Schwierigkeit mit dem Chatten keine Rolle, gleichwohl ist die Praxis auch dort Thema. ${ }^{11}$ Die Mitglieder der Gruppe elaborieren zunächst wechselseitig in ihren Einrichtungen populäre adoleszente Computermedienpraxen und klären so, inwieweit sie über strukturidentische Erfahrungen verfügen. Schliesslich eröffnet ihnen das Chatten ein allen vertrautes Thema, anhand dessen sich die Computermedienpraxen weiter ausführen lassen. Die folgende Sequenz illustriert, dass zwischen den Orientierungsmustern der Jugendarbeiter/innen auch Differenzierungen existieren, die in der Gruppendiskussion zum Tragen kommen und die Spannbreite der Orientierungsmuster sichtbar machen.

Df: Also, was mir auf jeden Fall in der Einrichtung aufgefallen ist, dass die Jungs (1) öfter und länger chatten, also auf Jahre mässig, ne. Die Mädchen sagen wirklich nach 'ner zeit, jetzt ist gut, so nach ein, zwei Jahren wollen sie nicht mehr. Aber die Jungs, die chatten auch noch mit zwanzig

$\mathrm{Nm}$ : Die lügen auch mehr finde ich

Df: Total, total. Das, die, genau, die spielen da Theater damit "@ ( 3 ) a "

Nm: Ja ja, auf alle Fälle. Die toben sich da richtig aus (3) Das ist

Dm: Also, ich find's ganz, ganz sinnvoll auch dieses Medium (1) Chat auch in dem Fall für pädagogische Arbeit zu nutzen, ne

Df

Dm: Manche haben die besten Gespräche sozusagen einfach, weil sie manchmal überhaupt nicht wissen sozusagen, wie sie Mädchen ansprechen sollen

Df: Echt jetzt?

Dm: Und, ähm, da

Af : $\quad \square J a ~ k l a r$

Df: Ja, die Mädchen ja auch gegenüber den Jungs

Dm: da über dieses Medium, da kommt tatsächlich auch ein Gespräch in Gang. Also, ich sitz daneben, und was soll ich ' $n$ jetzt schreiben? Sobald mal 'ne Antwort kommt. Das ist nett zu sehen eigentlich, weil das ganze Machogehabe fällt ab in dem Moment sozusagen. Sie sind so klein mit Hut und ja, das würden sie sich in der Disco nicht trauen, sagen wir's mal so

Df : Mhm

11 Die Gruppendiskussion wurde im Sommer 2004 geführt. Die Gruppe bestand aus zwei Frauen und zwei Männern im Alter zwischen (Anfang) 30 und (Ende) 40 Jahren; alle verfügten über mehrjährige medienpädagogische Arbeitserfahrungen. 


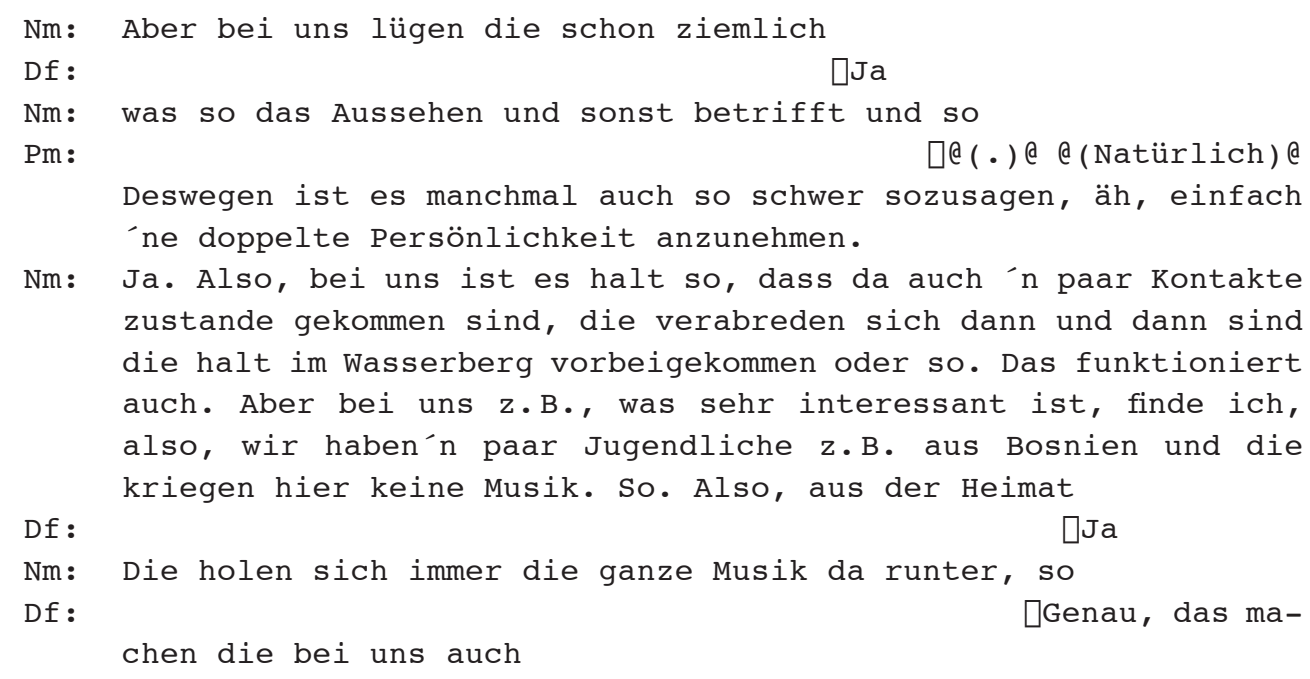

Doris weist als erste auf geschlechtsspezifische Unterschiede der Chatnutzung hin, die ihr in ihrer Einrichtung aufgefallen sind. Im Zentrum ihrer Wahrnehmung steht die Beobachtung, dass die Jungen im Zeitverlauf länger und häufiger chatten würden als die Mädchen. Letztere verlören nach ca. ein bis zwei Jahren das Interesse an der Praxis, während die Jungen auch noch im Alter von 20 Jahren chatten würden. Diese entwicklungstypische Differenzierung ist an das Lebensalter der Jugendlichen geknüpft. Für die Mädchen ist das Chatten nur für eine relativ kurze Zeitperiode interessant, während die Relevanz bei den Jungen wesentlich länger anhält. Worauf dieser Unterschied zurückzuführen ist, bleibt offen. Auch Norbert stellt beim Chatten geschlechtsspezifische Unterschiede fest. Die Jungen würden auch häufiger lügen als die Mädchen. Die Aussage impliziert, dass er entweder Einblick in die Kommunikation der Jugendlichen nimmt oder dass er im Zuge von Anschlusskommunikation etwas über die Inhalte der Kommunikation erfährt. Doris verfügt über strukturidentische Erfahrungen, bestätigt Norberts Feststellung und betont die Intensität und Reichweite der Praxis. Es scheint, als würden die Jungen den Chat nahezu ausschliesslich dazu nutzen, Anderen falsche Tatsachen vorzuspiegeln. Doris elaboriert das Phänomen weiter, indem sie darauf hinweist, dass die Jungen das Medium auch nutzen, um damit Theater zu spielen. Das Theaterspielen verweist auf Prozesse der Milieubildung: Zum einen nutzen die jungen Männer das Medium für eine spielerische Inszenierung. Führt man diese Beobachtung weiter, gelangt man zu einem Modus, mittels dem die jungen Männer biografische Orientierungen im Zuge der entwicklungstypischen Suche nach neuen Milieuzusammenhängen erproben können (Bohnsack 1998a: 123f). Ausserdem steht das Theater synonym für Getue oder Aufregung. Somit verweist diese Form der Inszenierung abermals auf aktionistische Praxen, die von hoher Relevanz für die Prozesse der Milieubildung sind und den Suchbewegungen einen Namen geben 
(s. o.). In diese Richtung zeigt auch die weitere Elaborierung der Praxis durch Norbert. Die jungen Männer würden den Chat auch nutzen, um sich «richtig» auszutoben. Die Charakterisierung der Praxis als ausgelassen und sich verausgabend verweist ebenfalls auf die aktionistischen Anteile der Praxis, die hier in den virtuellen Raum ausgedehnt werden (vgl. auch Welling/Brüggemann 2004: 26).

Mit dem Hinweis, dass er es sinnvoll fände, das Medium Chat auch für die pädagogische Arbeit zu nutzen, greift Dirk die, in der Diskussion bereits zuvor aufgeworfene pädagogische Nutzbarmachung der Praxis erneut auf. Manche der Jungen hätten dabei die besten Gespräche, weil sie überhaupt nicht wüssten, wie sie ein Mädchen ansprechen sollen. Mit der Erprobung der heterosexuellen Beziehungsanbahnung benennt Dirk zugleich ein weiteres zentrales und bildungsmilieuübergreifend wirksames Motiv der Chatpraxis (vgl. auch Schäffer 2003: 150-157). Da viele Jungen nur wenig Erfahrung mit der erfolgreichen, d. h. auf Perspektivenreziprozität angelegten verbalen Interaktion mit Mädchen haben, bietet ihnen der Chat aufgrund seiner distanzschaffenden Qualität und seiner Anonymität eine sichere Basis für solche Erprobungen. Doris scheint, wie ihre Nachfrage illustriert, von dieser Erfahrung überrascht zu sein, die aber umgehend von Adine kommunikativ validiert wird und offensichtlich über ähnliche Erfahrungen wie Dirk verfügt. Dirk fährt fort, dass diese Medienpraxis neben der Kommunikation zwischen Jungen und Mädchen überraschenderweise auch als Katalysator für die Kommunikation zwischen dem Jugendarbeiter und den Jugendlichen dient. Auf Basis des fallübergreifenden Vergleichs mit der Gruppe Traube, in der darauf hingewiesen wird, dass die Jugendlichen nicht wollen, dass die Jugendarbeiter/innen an der Chat-Kommunikation teilhaben (s. o.), wird die Überraschung nachvollziehbar. Im vorliegenden Fall lassen die Jugendlichen nicht nur die Nähe des Pädagogen zu, sondern erbitten auch seinen Rat. Dirk findet es sympathisch («nett»), zu beobachten, wie das "ganze Machogehabe» abfalle und die Jugendlichen «so klein mit Hut» seien, wenn sie eine Antwort erhalten. Die Praxis im Chat eignet sich also durchaus, geschlechtsstereotype Rollenbilder aufzubrechen und in Frage zu stellen. Anhand des negativen Gegenhorizontes der Diskothek, in der die Jugendlichen es nicht wagen würden, sich dergestalt zu verhalten, arbeitet Dirk den pädagogischen Wert der Praxis heraus. Im Gegensatz zum Chat ist es im Kontext der Peer-group und des gemeinsamen Diskobesuches nicht möglich, Schwächen und eigene Unsicherheiten einzugestehen. Der Pädagoge wird zum Ratgeber, der den Jugendlichen dabei helfen kann, mit solchen adoleszenztypischen Unsicherheiten in altersangemessener Weise umzugehen.

Norbert überzeugt der Hinweis von Dirk auf die pädagogische Nutzbarmachung des Chats nur eingeschränkt und differenziert dessen Proposition mit dem wiederholten Hinweis, dass die Jugendlichen in seiner Einrichtung häufig im Chat die Unwahrheit sagen würden. Doris verfügt über ähnliche Erfahrungen und verifiziert die Differenzierung. Lachend stimmt auch Dirk zu, das Gesagte stellt für ihn keinen 
Widerspruch dar. Stattdessen identifiziert er die Aussage als möglichen Grund, warum es den Jugendlichen gelegentlich schwer falle, eine «doppelte Persönlichkeit anzunehmen». Sie verändern die Charakteristika ihrer im Chat präsentierten Persönlichkeit so lange, bis sie nur noch so wenig mit der eigenen Person zu tun hat, dass man von zwei Persönlichkeiten sprechen kann. Wird diese Praxis über einen längeren Zeitraum verfolgt, stellt sie zum einen eine kognitive Herausforderung dar, indem die frei gestaltete Persönlichkeit bespielt wird, ohne sich beispielsweise in Widersprüche zu verstricken.

Trotz Dirks Erwiderung geht Norbert nicht weiter auf die Anschlussfähigkeit des Chattens für die intergenerationelle Praxis ein. Sie hat für ihn offensichtlich keine berufsbiografische Relevanz. Stattdessen eröffnet er mit einer weiteren Proposition eine neue Facette der Handlungspraxis. Das Chatten habe auch zur Folge, dass die Jugendlichen Kontakte anbahnen und diese in eine Praxis unter Anwesenden im Jugendzentrum überführen. Das, so Norbert, würde auch ‘funktionieren`. Im Sinne einer Funktion erhält dieser Teil des Chatten einen abstrahierenden Charakter, der insofern auch zum Betrieb des Gesamtsystems «Jugendzentrum» beiträgt, wenn auf diesem Wege neue Besucher/innen den Weg in die Einrichtung finden. Das Medium trägt damit letztlich auch zur Legitimationssicherung der Jugendeinrichtung bei, die auch an die Zahl der Besucher/innen gebunden ist. Daneben identifiziert Norbert eine weitere Computermedienpraxis der Jugendlichen, die er «sehr interessant», d. h. reizvoll findet und die seine Aufmerksamkeit erregt. Ein Teil der Besucher/innen verfügt über einen Migrationshintergrund und nutzt den Internetzugang im Jugendzentrum, um sich Musik aus ihrem oder dem Herkunftsland ihrer Eltern herunterzuladen, da sie diese Musik offensichtlich in Deutschland nicht käuflich erwerben können. Diese Form der Nutzung ist klar zweckrational und das ihr zugrunde liegende Motiv problemlos erkennbar, sodass die Sinnhaftigkeit der Handlungspraxis für den Jugendarbeiter, im Gegensatz zum Vorspielen falscher Tatsachen (s. o.) offensichtlich ist. Abermals ist es Doris, der die beschriebene Praxis auch aus ihrer Einrichtung bekannt ist. Erneut wird die weit reichende Strukturidentität der berufsbiografischen Erfahrungen der Jugendarbeiter/innen deutlich.

Neben solchen Handlungspraxen, die ohne das Zutun der Jugendarbeiter/innen auskommen bzw. in deren Verlauf intergenerationelle Bildungsprozesse vor allem im Verlauf von Anschlusskommunikation zustande kommen können, bilden Projekte eine der tragenden Säulen der Jugendarbeit. ${ }^{12}$ Auch in der medienpädago-

12 Der Begriff der intergenerationellen Bildungsprozesse ist grundlagentheoretisch und empirisch von Schäffer fundiert worden. Er bezeichnet damit solche Prozesse, «die sich auf ein Reflexivwerden des in diesen fundamentalen Lern- und Aneignungsprozessen erworbenen, generationsspezifischen handlungspraktischen Wissens beziehen» (Schäffer 2003: 213). Zu solchen Prozessen kommt es, «wenn generationsspezifische konjunktive Erfahrungsräume im Modus der Begegnung reflexiv relationiert werden. Dieser Prozess der reflexiven Relationierung konjunktiver Erfahrung wird immer dann angestossen, wenn durch Begegnungen zwischen konjunktiven Erfahrungsräumen habituelle Wissensbestände in Frage gestellt werden, wenn also ein unproblematisches (Weiter so) durch Dif- 
gischen Arbeit gilt die Projektarbeit vielerorts als 〈Königsweg〉. Auch die beiden Frauen aus der Gruppe Mango haben in der Vergangenheit verschiedene Projekte durchgeführt. ${ }^{13}$ Dazu gehörte u. a. die Erstellung eigener Internetseiten durch die Jugendlichen von der die Jugendarbeiterin Nora in der folgenden Sequenz berichtet (Passage Medienpädagogische Projektarbeit).

Nf: Dann war die Homepage fertig, haben sie natürlich nicht weiter irgendwie verfolgt oder gepflegt oder so

Cf :

$\mathrm{Nf}$ :

$\mathrm{Nf}$ :

Cf :

$\mathrm{Nf}:$

Cf : $\mathrm{Nf}:$

$\mathrm{Nf}:$

Cf :

$\mathrm{Nf}:$

$\mathrm{Cf}$ :

$\mathrm{Nf}$ : $\square$ Nein

Hauptsache fertig @(.)@ Gut ist, haben dann vielleicht noch zwei, drei Mal draufgeguckt. Die wissen ja auch gar nicht, was sie damit machen sollen, letztlich, ne

$\square$ Also, bei uns haben sie sie nur benutzt irgendwie, das war dann der totale Hit als sie ' ne Digitalkamera irgendwie immer wieder leihen konnten für die Homepagekurse. Dann haben sie die ganzen Fotos von sich, also, das war geil, das hat spass gemacht. Dann haben sie riesige Foto-Sessions gemacht, haben irgendwie echt irgendwie lange rumexperimentiert, welche Fotos sie dann auf die Seite stellen um dann, in 'nem chat, wenn jemand sie nach einem Bild fragt, sagen zu können, hey, da ist es. So, muss ich dir nicht schicken, sondern ist im Netz

da kannst du mich angucken

$\square \mathrm{Ja}$

Das war der einzige Sinn, wofür es diese Homepages gegeben hat. Wo ich dann dachte irgendwie, na super, ob ich das irgendwie, also, ob das mein pädagogisches ziel sein kann irgendwie

Ich dachte immer, oh man, jetzt liest jeder im Super-Chat, dass man irgendwie @(.)@, oh, ey, ich dachte so, ei, jei, jei

$\square$ e ( . ) @

a(Und ich bin die Schuldige) a. Das, das sind so Momente, wo ich dann mal denke so, ah, vielleicht ist das Konzept nicht so richtig aufgegangen

$\square \mathrm{Mhm}$

Ich habe die Dimensionen nicht beachtet

$\square \mathrm{Mhm}$

ferenzerfahrungen nicht mehr ohne weiteres möglich ist» (ebd.: 224, H.i.O.). Ebenfalls angesprochen ist in diesem Zusammenhang das Miteinandersein in leiblich-seelischer Hinsicht, das nicht wie im argumentativen Dialog auf Prozesse der sprachlich vermittelten Kommunikation und deren rationalistische Implikationen eingegrenzt ist (ebd.: 221).

13 Die Gruppendiskussion wurde im Sommer 2004 geführt. Die Gruppe bestand nur aus diesen beiden Personen im Alter zwischen (Ende) 30 und (Mitte) 40 Jahren; wie die Mitglieder der Gruppe Kirsche verfügten die beiden Personen ebenfalls über mehrjährige medienpädagogische Arbeitserfahrungen. 
Mit der Fertigstellung der Homepage endet jedes weitere Interesse der Mädchen in puncto Nutzung und Pflege des Medienprodukts. Für Nora ist dieses Desinteresse selbstverständlich («natürlich») und erklärt das Handeln der Jugendlichen, denn letztlich wüssten sie nicht, was sie mit einer eigenen Internetseite anfangen sollen. Das Produkt wird zum Selbstzweck, dass sich die Jugendlichen dennoch an seiner Produktion beteiligen, verweist auf ihre Prozessorientierung sowie ihr Interesse an einer Verwicklung in Aktivitäten unabhängig von deren Gegenstand. Ansonsten würden sie sich kaum an einem Projekt beteiligen, dessen Produktergebnis keine biografische Relevanz für sie besitzt.

Dass solche Internetseiten durchaus eine biografische Relevanz haben können, die eine längerfristigere Nutzung nach sich zieht, illustriert das Beispiel von Christine, mit dem sie die Thematik weiter elaboriert. Während eines Homepageworkshops wird den Teilnehmerinnen eine Digitalkamera zur Verfügung gestellt, mit der sie Porträts anfertigen, um diese auf einer eigenen Internetseite zu publizieren. Um zu einer zufrieden stellenden Bildauswahl zu gelangen, veranstalten die Mädchen «riesige Fotosessions» und experimentieren lange herum. Diese Praxis entspricht der bei vielen Mädchen populären Darstellung am Körper, die im vorliegenden Fall mit Hilfe der Computermedien erfolgt und von hoher Interaktivität und Intensität charakterisiert ist. Sie erproben sich in vielfältiger Form, bis die Darstellungen ihren Vorstellungen von sich selbst entsprechen. Die digitale Fotografie erweist sich als begeistert angenommenes Hilfsmittel für die im Verlauf der adoleszenten Persönlichkeitsentwicklung bedeutsame Ästhetisierung des eigenen Körpers (Bimschas/Schröder 2003: 108).

Die besten Fotos werden auf den Internetseiten platziert und dienen in Kombination mit diesem Medium der Verfolgung eines Motivs, das der Internetseite eine eng bemessene, zweckrational und instrumentalisierend begründete biografische Relevanz verleiht. Werden die Mädchen jetzt beim Chatten nach einem Bild von sich gefragt, müssen sie es nicht mehr per E-Mail verschicken, sondern können auf ihre Internetseite verweisen. Einen darüber hinausgehenden Nutzen des Mediums für die Mädchen kann die Pädagogin nicht entdecken. Obgleich die jungen Frauen sich intensiv mit dem Anfertigen von Fotos auseinandergesetzt haben und gelernt haben, wie man eine Internetseite erstellt, um darauf Fotos zu veröffentlichen, ist Christine mit dem Verlauf des Projektes nicht zufrieden und fragt sich, ob es mit der pädagogischen Zielstellung ihrer Arbeit vereinbar ist. Die Handlungspraxis der Mädchen wirft einen Konflikt mit den der Arbeit zugrunde liegenden professionellen berufsbiografischen Orientierungen auf und lässt sich nicht ohne weiteres mit diesen in Übereinstimmung bringen. Dabei handelt es sich aber nicht um medienpädagogische, sondern ausschliesslich um pädagogische Ziele. Die Medienpädagogik wird im Sinne ihres Status als Querschnittsaufgabe den pädagogischen Intentionen der Arbeit nachgeordnet bzw. werden die Medien von den 
Pädagogen/-innen als Mittel zum Zweck eingesetzt, um bestimmte pädagogische Ziele zu erreichen.

Es bereitet Christine Unbehagen, dass nun jeder im Chat Fotos der jungen Frauen anschauen könne und sie die Schuldige sei. Sie trägt die Verantwortung für das Tun der jungen Frauen und impliziert gleichzeitig ein daraus rührendes Vergehen oder Unrecht. Es stellt sich in diesem Sinne die Frage, ob sie z. B. mit ihrer Praxis Anforderungen des Persönlichkeits- und/oder Jugendschutzes, die darauf abzielen, Jugendliche vor potenziellen Gefahren der Internetnutzung zu schützen, bereits verletzt hat oder zumindest das Risiko besteht, eine solche Verletzung zu begünstigen. Als zentraler Gegensatz erweist sich in diesem Fall der gleichzeitige Wunsch, den Kindern und Jugendlichen möglichst weit reichende Freiheiten im Zuge der Subjektbildung (Scherr 2003) einzuräumen, sodass das medienpädagogische Handeln in ein Spannungsverhältnis zwischen dem Wunsch Freiheiten zu ermöglichen und Verantwortung übernehmen zu müssen, eingebettet ist, das ebenfalls typisch für die medienpädagogische Praxis ist.

Das angesprochene Beispiel lässt sich auf weitere Situationen des pädagogischen Alltags übertragen, da es immer wieder solche «Momente» gebe, in denen Christine denkt, dass ihr Konzept vielleicht nicht richtig aufgegangen sei. Es ist fraglich, ob der ihrem Projekt zugrunde liegende Handlungsentwurf bzw. -plan richtig war und ob sie vielleicht einen Fehler gemacht hat. Daneben vermutet sie, dass sie «die Dimensionen nicht beachtet» habe. Dimensionen als Ausdehnung oder Abmessung deuten dahin, dass unberücksichtigt blieb, welche möglichen biografischen Relevanzen eine Internetseite für die Mädchen haben könnte und inwieweit sie damit Praxen begünstigt, die nicht ihren pädagogischen Vorstellungen entsprechen. Das Spannungsverhältnis zwischen dem Wunsch danach, den Jugendlichen möglichst viel Freiheiten zu gewähren, um möglichst vielfältige Prozesse der Milieuund Subjektbildung zu ermöglichen, und der von den Jugendarbeitern/-innen zu übernehmenden Verantwortung wird an diesem Beispiel gut deutlich und markiert zugleich ein weiteres zentrales Typologieelement der medienpädagogischen Praxis. Die Übernahme von Verantwortung ist in den professionellen Orientierungsrahmen der Jugendarbeiter/innen und den die medienpädagogische Arbeit mitbestimmenden Orientierungsschemata verankert, am deutlichsten in gesetzlichen Bestimmungen, wie denen des Jugendschutzes, die in den Jugendeinrichtungen durchzusetzen oder einzuhalten sind. Andere Anforderungen sind unbestimmter und interpretierungsbedürftiger. So liegt z. B. der Aufforderung Medienkompetenz zu vermitteln kein einheitliches Verständnis darüber zugrunde, was es dabei genau zu vermitteln gilt. Solche Vorgaben verschmelzen mit den individuellen und kollektiven biografischen Orientierungen der Pädagogen/-innen zu den der medienpädagogischen Praxis zugrunde liegenden Orientierungsmustern. Gleichzeitig wird anhand dieses Abschnittes die Unbestimmtheitsdimension nicht formalisierter Bildungsprozesse deutlich, die durch ihre Ergebnisoffenheit charakterisiert sind 
(Lindner 2003). Neben Chancen, die geeignet sind, gängige Muster der Selbstund Weltauslegung der Jugendlichen in Frage zu stellen und Wandlungsprozesse anzustossen, bergen sie auch Risiken, die von den Jugendarbeitern/-innen abgewogen und im Zuge der Arbeit adäquat berücksichtigt werden müssen.

\section{Diskussion und Ausblick}

Anhand von Beispielen aus drei verschiedenen Gruppendiskussionen mit Jugendarbeiter/innen habe ich exemplarisch gezeigt, wie sich mit Hilfe der dokumentarischen Methode Orientierungsmuster rekonstruieren lassen, die dem Umgang mit den digitalen Medien in der Offenen Jugendarbeit zugrunde liegen. Auf diesem Wege lässt sich ein empirisch fundierter Beitrag zur medienpädagogischen Professionsforschung leisten. Wie in Kapitel 2 erörtert, ist die Vergleichsgruppenbildung bzw. komparative Analyse von zentraler Bedeutung für das rekonstruktive Verfahren. Nur auf diesem Wege lassen sich die Gemeinsamkeiten und Unterschiedlichkeit der Orientierungsmuster sowie daraus rührende professionelle Handlungspraxis herausarbeiten. Dabei ist nicht nur von Bedeutung, worüber die Personen in den verschiedenen Gruppen sprechen, sondern auch welche Themen für sie nicht relevant sind. Die umfassende Fremdheit gegenüber dem Chatten ist z. B. nur Thema in der Gruppe Traube, in den Gruppen Kirsche und Mango taucht sie nicht auf. Auch das anhand der Gruppe Traube rekonstruierte Spannungsverhältnis zwischen dem Wunsch nach Sicherheit und Ordnung auf der einen und der Unvorhersehbarkeit der Computermedienpraxis sowie der Spontaneität der adoleszenten Handlungspraxis auf der anderen Seite, spielt in diesen beiden Gruppen keine Rolle mehr. Insofern ist dieses Typologieelement medienpädagogischer Praxis nur charakteristisch für Pädagogen/-innen, die am Anfang ihrer medienpädagogischen Praxis stehen und lediglich über begrenzte Erfahrungen im Umgang mit dem Computer verfügen.

Mit zunehmender Erfahrung tritt an die Stelle von Sicherheit und Ordnung der Wunsch der Jugendarbeiter/innen nach einer verbindlicheren Handlungspraxis der Jugendlichen. Dieser steht aber im ständigen Widerspruch zur Spontaneität der adoleszenten Handlungspraxis und der Freiwilligkeit der Teilnahme als zentralem Strukturprinzip in der Offenen Jugendarbeit (vgl. genauer Welling 2007). Das dritte zentrale Typologieelement basiert auf dem Gegensatz zwischen dem Wunsch, den Jugendlichen möglichst umfassende Freiheiten zu ermöglichen und der von den Pädagogen/-innen für die Handlungspraxis der Jugendlichen zu übernehmenden Verantwortung. Die Gruppe Mango steht stellvertretend für dieses Typologieelement. Anhand der Sequenz aus der Diskussion mit dieser Gruppe wird auch deutlich, dass die medienpädagogische Ziele bisweilen gegenüber den originär pädagogischen Zielen im Sinne von Orientierungsschemata von nachgeordneter Relevanz sind. Die Medienarbeit wird dann Mittel zum Zweck, um bestimmte pädagogische Ziele zu erreichen, die ohne Medienbezug auskommen. Diese Skizzie- 
rung unterstreicht auch, dass das Gruppendiskussionsverfahren geeignet ist, um die von Hugger kritisierte reduktive Tendenz einer Idealtypenbildung zu überkommen und durch eine empirisch fundierte Typenbildung zu ersetzen (vgl. Kap. 1). Der fallübergreifende Vergleich eröffnet einen differenzierten Blick auf die variierenden Voraussetzungen für die Enaktierung intergenerationeller Bildungsprozesse im Verlauf der Chatpraxis. Im Fall der Gruppe Traube kommt es dabei aufgrund ihrer umfassenden Fremdheit gegenüber der Praxis am ehesten zu Verlaufkurven und damit zu Prozessen der Verengung und Abschottung. Dagegen zeigt die Gruppe Kirsche, wo sich im Verlauf der Praxis Möglichkeiten für die Initiierung intergenerationeller Bildungsprozesse eröffnen, wenn die Pädagogen/-innen mittels Anschlusskommunikation Wandlungsprozesse bei den Jugendlichen anstossen können. Die Gruppe Mango wiederum veranschaulicht, dass die Pädagogen/innen mit Hilfe des gezielten Medieneinsatzes und der Verbindung mit bei den Jugendlichen ohnehin populären Medienpraxen Situationen schaffen können, die Bildungspotenzial besitzen. Eingeschränkt werden solche Möglichkeiten vor allem durch divergierende Orientierungsschemata, die die Umsetzung der Handlungspraxen mitbestimmen. Der Vergleich über alle drei Gruppen hinweg macht deutlich, dass die Zweckrationalität der adoleszenten Computermedienpraxen ein wichtiges Basiskriterium für die positive Beurteilung der Praxen ist und grossen Anteil an den berufsbiografischen Orientierungen der Pädagogen/-innen hat. Gleichwohl muss dabei gewährleistet sein, dass korrespondierende Handlungspraxen im Einvernehmen mit den dominanten Orientierungsschemata der Jugendarbeiter/ innen erfolgen (s. o.).

Die empirischen Beispiele in Kapitel 3 haben auch anschaulich gezeigt, wie die kollektiven Orientierungsmuster der Jugendarbeiter/innen sowie deren Grenzen erst durch die Spezifik der Erhebungssituation für die empirische Analyse zugänglich werden. Die Möglichkeit der Diskussionsteilnehmer/innen jederzeit Bezug aufeinander nehmen zu können und Beiträge der Mitdiskutierenden kommentieren, elaborieren und differenzieren zu können, erweist sich als ein zentraler Vorteil der durch das Gruppendiskussionsverfahren geschaffenen Erhebungssituation. Auch in Gruppeninterviews, die häufig mit Gruppendiskussionen verwechselt werden, kommt eine solche interaktive Bezugnahme der Sprecher/innen nicht zu Stande, ganz zu schweigen von Einzelinterviews. Erst durch die gegenseitige Bezugnahme der Teilnehmer/innen sowie der Verifizierung der Beschreibungen und Erzählungen einzelner Gruppenmitglieder wird in den drei Gruppen deutlich, wie weiterführend die strukturidentischen Erfahrungen und die gemeinsamen berufsbiografischen Orientierungen der Pädagogen/-innen sind. Besonders gut deutlich ist das anhand der Gruppe Traube geworden. Im Verlauf der Diskussion beginnen sich die beiden Jugendarbeiterinnen Doris und Brigitte zu steigern und sprechen in der Wortüberlappung fast mit einer Stimme, wenn sie ihre Fremdheit gegenüber der im Chat benutzten Kunstsprache formulieren. Andererseits zeigen insbesondere 
die Differenzierungen auf, wo die individuellen Grenzen und Unterschiede der berufsbiografischen Orientierungen verlaufen.

Das Gruppendiskussionsverfahren erweist sich auch als nützlich, um der ebenfalls in Kapitel 1 angesprochenen Relevanz des Wechsels zwischen Aktion und Reflexion gerecht zu werden. Denn es sind genau jene Wirklichkeitskonstruktionen, die im Verlauf der Alltagspraxis erfolgen, die Gegenstand der Interpretation sind. Gleichzeitig haben wir bei der Durchführung von Gruppendiskussionen mit Professionellen die Erfahrung gemacht, dass viele der Teilnehmer/innen darin eine willkommene Gelegenheit sehen, ihre medienpädagogischen Erfahrungen mit anderen zu vergleichen und zu reflektieren. Denn in ihrem beruflichen Alltag haben sie dazu häufig keine Gelegenheit, da sie dort oft als einzige medienpädagogisch tätig sind. Vereinzelt wurde dabei auch der Wunsch formuliert, das berufliche Handeln systematisch zu reflektieren (Welling/Brüggemann 2004: 81-83). Gruppendiskussionen können damit u. U. auch einen Beitrag zur Entwicklung einer reflexiven Professionalisierung leisten, wie sie ebenfalls in Kapitel 1 angesprochen wurde, und die von verschiedener Seite immer wieder als zentrale Voraussetzung einer gelingenden Sozial- und damit auch der Medienpädagogik angemahnt wird (Bimschas/Schröder 2003, Schweppe 2003: 148, Hafeneger 2007: 19).

Trotz des offensichtlichen Nutzens des Gruppendiskussionsverfahrens für die medienpädagogische Professionsforschung können damit natürlich nicht beliebige Fragestellungen bearbeitet werden. So eignet sich das Verfahren z. B. nicht für die Untersuchung biografischer Aspekte der professionellen Praxis, da Beiträge nicht individuell ausgewertet werden, sondern die Individuen als Repräsentanten der jeweiligen Gruppe angesehen werden. Das Verfahren eignet sich auch nicht, um subjektive Intentionen der Gruppenmitglieder zu erfassen, was grundlagentheoretisch darauf zurückzuführen ist, dass die einer Äusserung zugrunde liegenden Motive von den Interpretierenden nur vermutet oder unterstellt werden können. Ähnliches gilt für die Untersuchung von Handlungspraxen, da dem Gespräch über die Handlungspraxis das Typische der Praxis fehlt. Gemeint ist damit die Involviertheit in die Praxis und die fehlende Möglichkeit, einfach aus ihr aussteigen zu können (Loos/Schäffer 2001: 39ff).

Daher gilt es im Vorfeld einer Untersuchung zu überprüfen, inwieweit das Gruppendiskussionsverfahren geeignet ist, den interessierenden Untersuchungsgegenstand bzw. die zu bearbeitende Fragstellung in methodisch-methodologischer Weise adäquat zu bearbeiten. Das ist aber nicht nur eine Entscheidung für oder gegen die Methode, sondern adressiert auch die Frage, inwieweit das Gruppendiskussionsverfahren im Kontext der Prämisse der Methodentriangulation sinnvoll mit anderen Erhebungs- und Auswertungsmethoden verbunden werden kann. In dem Forschungsprojekt, das diesem Beitrag zugrunde liegt, wurde das Verfahren beispielsweise mit der teilnehmenden Beobachtung kombiniert. Sie diente dabei erstens dazu, den Zugang zum Feld herzustellen. Zweitens konnte so ein erster 
Eindruck davon gewonnen werden, wie die Jugendlichen in den Jugendeinrichtungen mit den Jugendarbeiter/innen interagieren und welche Rollen die digitalen Medien dabei spielen. Drittens liefern zufällig vor Ort gemachte Beobachtungen alläglicher Handlungspraxen, die in den Gruppendiskussionen nicht thematisiert wurden, zusätzliche Hinweise zu den untersuchten Milieus und bereicherten den Forschungsprozess. Viertens lässt sich mit Hilfe der teilnehmenden Beobachtung Verhalten in seinem unmittelbaren Entstehungszusammenhang erfassen sowie dokumentieren und die Beobachteten können teilweise zu einem späteren Zeitpunkt dazu befragt werden (Lüders 2000, Kawulich 2005, Friebertshäuser 1997).

Schliesslich sei noch darauf hingewiesen, dass es sich beim Gruppendiskussionsverfahren um ein textanalytisches Verfahren handelt. Im Rahmen der medienpädagogischen Arbeit spielen gleichwohl auch die eingesetzten Medien und die unter ihrer Zuhilfenahme erstellten Produkte und der weitere Umgang damit eine wichtige Rolle. Hier besteht im Zuge der medienpädagogischen Professionsforschung ein offensichtlicher Entwicklungsbedarf, diese Produkte in den Forschungsprozess zu integrieren. Als anschlussfähig könnten sich in diesem Kontext, Teile der in der letzten Zeit verstärkt unternommenen Anstrengungen bezüglich der Weiterentwicklung visueller Methoden erweisen (Marotzki/Niesyto 2006, Friebertshäuser u. a. 2007). Die Verbindung solcher Verfahren mit textanalytischen Methoden erscheint viel versprechend und könnte zur Erschliessung neuer Erkenntnisdimensionen beitragen, um vor allem noch mehr darüber zu erfahren und zu verstehen, wie die Adressaten medienpädagogischer Arbeit - und um die geht es ja hier in letzter Konsequenz - die unterschiedlichen Medien einsetzen und welche Auswirkungen das auf die Veränderung ihrer Selbst- und Weltreferenzen als Kern eines qualitativen Verständnisses von Medienbildung hat. 


\section{Literaturverzeichnis}

Baacke, Dieter. Medienpädagogik. Tübingen: Niemeyer, 1997.

Bimschas, Bärbel; Schröder, Achim. Beziehungen in der Jugendarbeit: Untersuchung zum reflektierten Handeln in Profession und Ehrenamt. Opladen: Leske + Budrich, 2003.

Böhnisch, Lothar; Münchmeier, Richard. Wozu Jugendarbeit? Orientierungen für Ausbildung, Fortbildung und Praxis. Weinheim, München: Juventa, 1987.

Bohnsack, Ralf. Generation, Milieu und Geschlecht - Ergebnisse aus Gruppendiskussionen mit Jugendlichen. Opladen: Leske + Budrich, 1989.

Bohnsack, Ralf. Adoleszenz, Aktionismus und die Emergenz von Milieus: Eine Ethnographie von Hooligan-Gruppen und Rockbands. Zeitschrift für Sozialisationsforschung und Erziehungssoziologie 17.1 (1997a): 3-18.

Bohnsack, Ralf. "Orientierungsmuster»: Ein Grundbegriff qualitativer Sozialforschung. Methodische Probleme der empirischen Erziehungswissenschaft. Hrsg. v. Folker Schmidt. Baltmannsweiler: Schneider, 1997b. 49-61.

Bohnsack, Ralf. Milieu als konjunktiver Erfahrungsraum: Eine dynamische Konzeption von Milieu in empirischer Analyse. Die Räume der Milieus: Neue Tendenzen in der sozialund raumwissenschaftlichen Milieuforschung, in der Stadt- und Raumplanung. Hrsg. v. Ulf Matthiesen. Berlin: Edition Sigma, 1998a. 119-149.

Bohnsack, Ralf. «Milieubildung» - Pädagogisches Prinzip und empirisches Phänomen. Hrsg. v. Böhnisch, Lothar; Rudolph, Martin; Wolf, Barbara: Jugendarbeit als Lebensort. Weinheim u. München: Juventa, 1998b. 95-112.

Bohnsack, Ralf. Rekonstruktive Sozialforschung - Einführung in die Methodologie und Praxis. Opladen: Leske + Budrich, 2000.

Bohnsack, Ralf. Typenbildung, Generalisierung und komparative Analyse. Grundprinzipien der dokumentarischen Methode. Die dokumentarische Methode und ihre Forschungspraxis: Grundlagen qualitativer Forschung. Hrsg. v. Ralf Bohnsack, Iris Nentwig-Gesemann u. Arnd-Michael Nohl. Opladen, Leske + Budrich. 2001. 225-252.

Bohnsack, Ralf. Rekonstruktive Sozialforschung - Einführung in qualitative Methoden. Opladen: Leske + Budrich, 2003.

Bohnsack, Ralf. Standards nicht-standardisierter Forschung in den Erziehungs- und Sozialwissenschaften. Zeitschrift für Erziehungswissenschaft. Beiheft 4: Standards und Standardisierung in der Erziehungswissenschaft 7 (2005): 65-83.

Bohnsack, Ralf; Nentwig-Gesemann, Iris; Nohl, Arnd-Michael (Hrsg.). Die dokumentarische Methode und ihre Forschungspraxis: Grundlagen qualitativer Forschung. Opladen: Leske + Budrich, 2001 a.

Bohnsack, Ralf; Nentwig-Gesemann, Iris; Nohl, Arnd-Michael. Einleitung. Die dokumentarische Methode und ihre Forschungspraxis: Grundlagen qualitativer Forschung. Hrsg. v. Ralf Bohnsack, Iris Nentwig-Gesemann u. Arnd-Michael Nohl. Opladen, Leske + Budrich. 2001b. 9-24.

Bohnsack, Ralf; Nohl, Arnd-Michael. Exemplarische Textinterpretation: Die Sequenzanalyse der dokumentarischen Methode. Die dokumentarische Methode und ihre Forschungspraxis: Grundlagen qualitativer Forschung. Hrsg. v. Ralf Bohnsack, Iris Nentwig-Gesemann u. Arnd-Michael Nohl. Opladen, Leske + Budrich. 2001. 303-307.

Bohnsack, Ralf; Przyborski, Aglaja. Diskursorganisation, Gesprächsanalyse und die Methode der Gruppendiskussion. Das Gruppendiskussionsverfahren in der Forschungspraxis. Hrsg. v. Ralf Bohnsack, Aglaja Przyborski u. Burkhard Schäffer. Opladen: Barbara Budrich, 2006. 233-248. 
Bohnsack, Ralf; Przyborski, Aglaja; Schäffer, Burkhard (Hrsg.). Das Gruppendiskussionsverfahren in der Forschungspraxis. Opladen: Barbara Budrich, 2006a.

Bohnsack, Ralf; Przyborski, Aglaja; Schäffer, Burkhard. Einleitung: Gruppendiskussionen als Methode rekonstruktiver Sozialforschung. Das Gruppendiskussionsverfahren in der Forschungspraxis. Hrsg. v. Ralf Bohnsack, Aglaja Przyborski u. Burkhard Schäffer. Opladen: Barbara Budrich. 2006b. 7-22.

Bourdieu, Pierre. Die Praxis der reflexiven Anthropologie. Reflexive Anthropologie. Hrsg. v. Pierre Bourdieu, Loïc J. D. Wacquant. Frankfurt a. M.: Suhrkamp, 1996. 251-294.

Brüggemann, Marion; Welling, Stefan. "Sie können «Bremen for you» eingeben und in ihren Chat und das war's» - Zentrale Herausforderungen medienpädagogischen Handelns in offenen Bereichen der Jugendarbeit dargestellt am Beispiel des Chattens. Schöne neue Medienwelten? Konzepte und Visionen einer Medienpädagogik der Zukunft. Hrsg. v. Ingrid Volkmer u. Dieter Wiedemann. Bielefeld: Gesellschaft für Medienpädagogik und Kommunikationskultur, 2006. 162-175.

Buchen, Sylvia. PC/Interneterfahrungen von Schülerinnen einer katholischen Mädchenrealschule. Die Nutzungspraxis als funktionales Äquivalent für andere Handlungsmodi. Hrsg. v. Buchen, Sylvia; Helfferich, Cornelia; Maier, Maja. S. Gender methodologisch. Empirische Forschung in der Informationsgesellschaft vor neuen Herausforderungen. Wiesbaden: VS Verlag für Sozialwissenschaften. 2004. 67-87.

Buchen, Sylvia. Neue Medien und Habitusformen von Hauptschüler(inne)n: Zum Widerspruch zwischen männlichem Habitus und faktischem Wissen. Der Deutschunterricht LVIII.3 (2006). 87-92.

Buchen, Sylvia; Philipper, Ingeborg. Die Bedeutung neuer Medien im Leben männlicher und weiblicher Jugendlicher unterschiedlicher Schulformen: Wie können biografische und generationsspezifische Bildungspotentiale durch veränderte Lernarrangements in der Schule genutzt werden? MedienPädagogik 24.7.2002: 〈http://www.medienpaed. com/02-1/buchen_philipper1.pdf> [12.7.2006].

Buchen, Sylvia; Straub, Ingo. Die Bedeutung des Hacker-Topos für Hauptschüler in der Adoleszenz. Gender medienkompetent: Medienbildung in einer heterogenen Gesellschaft. Hrsg. v. Annette Treibel, Maja S. Maier, Sven Kommer, Manuela Welzel. Opladen: VS Verlag für Sozialwissenschaften, 2006a. 93-110.

Buchen, Sylvia; Straub, Ingo. Die Rekonstruktion der digitalen Handlungspraxis Jugendlicher als Theoriegrundlage für eine geschlechterreflexive schulische Medienbildung. MedienPädagogik 26.4.2006 (2006b) shttp://www.medienpaed.com/05-2/buchen_ straub05-2.pdf> [20.6.2006].

Combe, Arno; Helsper, Werner. Einleitung: Pädagogische Professionalität. Historische Hypotheken und aktuelle Entwicklungstendenzen. Pädagogische Professionalität: Untersuchungen zum Typus pädagogischen Handelns. Hrsg. v. Arno Combe, Werner Helsper. Frankfurt a. M.: Suhrkamp, 1996. 9-48.

Delmas, Naine; Scherr, Albert. Bildungspotenziale der Jugendarbeit: Ergebnisse einer explorativen empirischen Studie. Deutsche Jugend: Zeitschrift für Jugendfragen und Jugendarbeit 53.3 (2005): 105-109.

Friebertshäuser, Barbara. Feldforschung und teilnehmende Beobachtung. Handbuch qualitative Forschungsmethoden in der Erziehungswissenschaft. Hrsg. v. Barbara Friebertshäuser, Annedore Prengel. Weinheim u. München: Juventa, 1997. 503-534.

Friebertshäuser, Barbara; Felden, Heide von; Schäffer, Burkhard (Hrsg.). Bild und Text: Methoden und Methodologien visueller Sozialforschung in der Erziehungswissenschaft. Opladen: Barbara Budrich, 2007. 
Hafeneger, Benno. Professionsbilder und -merkmale in der Jugendarbeit/Pädagogik. Deutsche Jugend. Zeitschrift für Jugendfragen und Jugendarbeit 1 (2007): 13-20.

Hugger, Kai-Uwe. Medienpädagogik als Profession: Perspektiven für ein neues Selbstverständnis. München: kopaed, 2001.

Hugger, Kai-Uwe. Medienpädagogik zwischen Kompetenz und Profession. Beruf Medienpädagoge: Selbstverständnis - Ausbildung - Arbeitsfelder. Hrsg. v. Norbert Neuss. München: kopaed, 2003. 41-54.

Hugger, Kai-Uwe. Medienpädagogen im Beruf. Schöne neue Medienwelten? Konzepte und Visionen einer Medienpädagogik der Zukunft. Hrsg. v. Ingrid Volkmer u. Dieter Wiedemann. Bielefeld: Gesellschaft für Medienpädagogik und Kommunikationskultur, 2006. 136-149.

Kawulich, Barbara B. Participlant Oberservation as a Data Collection Method. Forum Qualitative Social Research 6.2 (2005): ‘http://www.qualitative-research.net/fqs-texte/2-05/052-43-e.htm> [2.12.2006].

King, Vera; Müller, Burkhard K. Adoleszenzforschung und pädagogische Praxis - Zu systematischen Reflexion von sozialen Rahmenbedingungen und Beziehungskonflikten in der Jugendarbeit. Adoleszenz und pädagogische Praxis. Bedeutungen von Geschlecht, Generation und Herkunft in der Jugendarbeit. Hrsg. v. Vera King, u. Burkhard K. Müller. Freiburg im Breisgau: Lambertus, 2000. 29-35.

Krüger, Heinz-Hermann; Kötters-König, Catrin; Pfaff, Nicolle; Schmidt, Ralf. Jugend, Demokratie und politische Bildung. Jugend und Demokratie - politische Bildung auf dem Prüfstand. Eine quantitative und qualitative Studie aus Sachsen-Anhalt. Hrsg. v. HeinzHermann Krüger, Sibylle Reinhardt, Catrin Kötters-König, Nicolle Pfaff, Ralf Schmidt, Adrienne Krappidel u. Frank Tillmann Opladen: Leske + Budrich, 2002. 11-42.

Kutscher, Nadia. Moralische Begründungsstrukturen professionellen Handelns in der Sozialen Arbeit: Eine empirische Untersuchung zu normativen Deutungs- und Orientierungsmustern in der Jugendhilfe. Dissertation an der Universität Bielefeld. (2003) «http://bieson.ub.uni-bielefeld.de/volltexte/2003/406/〉 [2.3.2007].

Kutscher, Nadia. Die Rekonstruktion moralischer Orientierungen von Professionellen auf der Basis von Gruppendiskussionen. Das Gruppendiskussionsverfahren in der Forschungspraxis. Hrsg. v. Ralf Bohnsack, Aglaja Przyborski u. Burkhard Schäffer. Opladen: Barbara Budrich, 2006. 190-201.

Lindner, Werner. Alles Bildung!? - Kinder und Jugendarbeit in der «Wissensgesellschaft». Kinder- und Jugendarbeit als Bildungsprojekt. Hrsg. v. Werner Lindner, Werner Thole u. Jochen Weber. Opladen: Leske + Budrich, 2003. 47-68.

Loos, Peter; Schäffer, Burkhard. Das Gruppendiskussionsverfahren. Opladen: Leske + Budrich, 2001.

Lüders, Christian. Beobachten im Feld und Ethnographie. Qualitative Forschung: Ein Handbuch. Hrsg. v. Uwe Flick, Ernst von Kardorff u. Ines Steinke. Hamburg: Rowohlt Taschenbuch Verlag, 2000. 384-401.

Mannheim, Karl. Beiträge zur Theorie der Weltanschauungsinterpretation. Wissenssoziologie. Neuwied: Luchterhand, 1964 (1921). 91-154.

Marotzki, Winfried. Entwurf einer strukturalen Bildungstheorie: Biographietheoretische Auslegung von Bildungsprozessen in hochkomplexen Gesellschaften. Weinheim: Deutscher Studien-Verlag, 1990.

Marotzki, Winfried; Niesyto, Horst (Hrsg.). Bildverstehen und Bildinterpretation: Methodische Ansätze aus sozialwissenschaftlicher, kunst- und medienpädagogischer Perspek- 
tive. Medienbildung und Gesellschaft 2. Wiesbaden: VS Verlag für Sozialwissenschaften, 2006.

Neuss, Norbert (Hrsg.). Beruf Medienpädagoge: Selbstverständnis - Ausbildung - Arbeitsfelder. München: kopaed, 2003.

Niesyto, Horst. Medienpädagogik und soziokulturelle Unterschiede. Langfassung einer Studie auf der Basis von Experten-Interviews in Baden-Württemberg und Rheinland-Pfalz. Ludwigsburg: Verlag Pädagogische Hochschule Ludwigsburg, 2004.

Nohl, Arnd-Michael. Bildung und Spontaneität. Phasen biographischer Wandlungsprozesse in drei Lebensaltern - Empirische Rekonstruktionen und pragmatische Reflexionen. Opladen: Barbara Budrich, 2006.

Oevermann, Ulrich. Theoretische Skizze einer revidierten Theorie professionalisierten Handelns. Pädagogische Professionalität. Untersuchungen zum Typus pädagogischen Handelns. Hrsg. v. Arno Combe u. Werner Helsper. Frankfurt a. M.: Suhrkamp, 1996. 70-182.

Pietrass, Manuela. Medienbildung. Handbuch Bildungsforschung. Hrsg. v. Rudolf Tippelt. Opladen: Leske + Budrich, 2002. 393-408.

Przyborski, Aglaja. Gesprächsanalyse und dokumentarische Methode: Qualitative Auswertung von Gesprächen, Gruppendiskussionen und anderen Diskursen. Opladen: VS Verlag für Sozialwissenschaften, 2004.

Rein, Antje von (Hrsg.). Medienkompetenz als Schlüsselbegriff: Theorie und Praxis der Erwachsenenbildung. Bad Heilbrunn: Deutsches Institut für Erwachsenenbildung, Verlag Julius Klinkhardt, 1996.

Schäffer, Burkhard. Das Gruppendiskussionsverfahren in erziehungswissenschaftlicher Medienforschung. Medien Pädagogik 23.3.2001: 〈http://www.medienpaed.com/01-1/ schaeffer1.pdf> [28.5.2007].

Schäffer, Burkhard. Generation - Medien - Bildung: Medienpraxiskulturen im Generationsvergleich. Opladen: Leske + Budrich, 2003

Schell, Fred. Bedeutung von Medienkompetenz als Bildungsaufgabe und inhaltliche bildungspolitische Zielsetzung. Medienkompetenz. Grundlagen und pädagogisches Handeln. Hrsg. v. Fred Schell, Elke Stolzenberg u. Helga Theunert. München: kopaed, 1999. 272-281.

Scherr, Albert. Jugendarbeit als Subjektbildung: Grundlagen und konzeptionelle Orientierungen jenseits von Prävention und Hilfe zur Lebensbewältigung. Kinder- und Jugendarbeit als Bildungsprojekt. Hrsg. v. Werner Lindner, Werner Tholeu. Jochen Weber. Opladen: Leske + Budrich, 2003. 33-45

Schütze, Fritz. Sozialarbeit als «bescheidene» Profession. Zur Logik professionellen Handelns in pädagogischen Feldern: Erziehen als Profession. Hrsg. v. Bernd Dewe, Wilfried Ferchhof u. Frank Olaf Radtke. Opladen: Leske + Budrich. 1992. 132-170.

Schütze, Fritz. Das Paradoxe in Felix' Leben als Ausdruck eines «wilden» Wandlungsprozesses. Lebensgeschichte als Text: Zur biographischen Artikulation problematischer Bildungsprozesse. Marek Czyzewski u.a. Weinheim: Deutscher Studien-Verlag, 1994. 13-60.

Schütze, Fritz. Schwierigkeiten bei der Arbeit und Paradoxien des professionellen Handelns. Ein grundlagentheoretischer Aufriss. Zeitschrift für qualitative Bildungs-, Beratungs- und Sozialforschung 1. (2000): 49-96.

Schweppe, Cornelia. Wie handeln Sozialpädagoglnnen? Rekonstruktionen der professionellen Praxis der Sozialen Arbeit. Qualitative Forschung in der Sozialpädagogik. Hrsg. v. Cornelia Schweppe. Opladen: Leske + Budrich, 2003. 145-165. 
Straub, Ingo. Neue Freunde durch neue Medien? Die Bedeutung computervermittelter Kommunikation für männliche Jugendliche. MedienPädagogik 12.10.2005: 〈http://www.medienpaed.com/05-2/straub1.pdf〉 [12.7.2006].

Straub, Ingo. Medienpraxiskulturen männlicher Jugendlicher: Medienhandeln und Männlichkeitskonstruktionen in jugendkulturellen Szenen. Wiesbaden: Deutscher Universitätsverlag, 2006.

Thole, Werner. Kinder- und Jugendarbeit: Eine Einführung. Weinheim u. München: Juventa, 2001.

Thole, Werner. «Wir lassen uns unsere Weltsicht nicht verwirren»: Rekonstruktive, qualitative Sozialforschung und Soziale Arbeit - Reflexionen über ein ambivalentes Feld. Qualitative Forschung in der Sozialpädagogik. Hrsg. v. Cornelia Schweppe. Opladen: Leske + Budrich, 2003. 43-65.

Tiefel, Sandra. Beratung und Reflexion: Eine qualitative Studie zu professionellem Beratungshandeln in der Moderne. Wiesbaden: VS Verlag für Sozialwissenschaften, 2004.

Walser, Karin. Professionelles Können in der Jugendarbeit. Adoleszenz und pädagogische Praxis: Bedeutungen von Geschlecht, Generation und Herkunft in der Jugendarbeit. Hrsg. v. Vera King u. Burkhard K. Müller. Freiburg im Breisgau: Lambertus, 2000. 251-268.

Welling, Stefan. Chatten, Gamen, Downloaden - eine kritische Auseinandersetzung mit den Potenzialen einer milieusensitiven computerunterstützten Jugendarbeit. Dissertation an der Universität Bremen, 2007.

Welling, Stefan; Brüggemann, Marion. Computerunterstützte Jugendarbeit und medienpädagogische Qualifizierung: Praxis und Perspektiven. Bremen: Institut für Informationsmanagement Bremen GmbH (ifib), 2004.

Wimmer, Michael. Zerfall des Allgemeinen - Wiederkehr des Singulären: Pädagogische Professionalität und der Wert des Wissens. Pädagogische Professionalität. Untersuchungen zum Typus pädagogischen Handelns. Hrsg. v. Arno Combe u. Werner Helsper. Frankfurt a. M.: Suhrkamp, 1996. 404-447.

Zacharias, Wolfgang. Zum Beispiel AG Inter@ktiv in München: Kommunale Netzwerk-Infrastrukturen - zwischen «Lebenswelt \& Internet» und zwischen «Sinn \& Cyber». Informelle Bildung online. Perspektiven für Bildung, Jugendarbeit und Medienpädagogik. Hrsg. v. Hans-Uwe Otto u. Nadia Kutscher. München u. Weinheim: Juventa, 2004. 167-187.

Zirfas, Jörg; Wulf, Christoph. Integration im Ritual: Performative Prozesse und kulturelle Differenz. Zeitschrift für Erziehungswissenschaft 4.2 (2001): 191-208. 